TRANSACTIONS OF THE

AMERICAN MATHEMATICAL SOCIETY

Volume 364, Number 12, December 2012, Pages 6187-6227

S 0002-9947(2012)05650-9

Article electronically published on July 10, 2012

\title{
HOMOGENIZATION OF ACCELERATED FRENKEL-KONTOROVA MODELS WITH $n$ TYPES OF PARTICLES
}

\author{
N. FORCADEL, C. IMBERT, AND R. MONNEAU
}

\begin{abstract}
We consider systems of ODEs that describe the dynamics of particles. Each particle satisfies a Newton law (including a damping term and an acceleration term) where the force is created by the interactions with other particles and with a periodic potential. The presence of a damping term allows the system to be monotone. Our study takes into account the fact that the particles can be different.

After a proper hyperbolic rescaling, we show that solutions of these systems of ODEs converge to solutions of some macroscopic homogenized HamiltonJacobi equations.
\end{abstract}

\section{INTRODUCTION}

The goal of this paper is to obtain homogenization results for the dynamics of accelerated Frenkel-Kontorova type systems with $n$ types of particles. The FrenkelKontorova model is a simple physical model used in various fields: mechanics, biology, chemistry, etc. The reader is referred to [4 for a general presentation of models and mathematical problems. In this introduction, we start with the simplest accelerated Frenkel-Kontorova model where there is only one type of particle (see eq. (1.2)). We then explain how to deal with $n$ types of particles (see eq. (1.6)). We finally present the general case, namely systems of ODEs of the following form (for a fixed $m \in \mathbb{N}$ ):

$$
m_{0} \frac{d^{2} U_{i}}{d \tau^{2}}+\frac{d U_{i}}{d \tau}=F_{i}\left(\tau, U_{i-m}, \ldots, U_{i+m}\right),
$$

where $U_{i}(\tau)$ denotes the position of the particle $i \in \mathbb{Z}$ at the time $\tau$. Here, $m_{0}$ is the mass of the particle and $F_{i}$ is the force acting on the particle $i$, which will be made precise later.

Note the presence of the damping term $\frac{d U_{i}}{d \tau}$ on the left hand side of the equation. If the mass $m_{0}$ is assumed to be small enough, then this system is monotone. We will make such an assumption, and the monotonicity of the system is crucial in our analysis.

We recall that the case of fully overdamped dynamics, i.e. for $m_{0}=0$, has already been treated in [10] (for only one type of particle).

Received by the editors June 30, 2009 and, in revised form, June 6, 2010.

2010 Mathematics Subject Classification. Primary 35B27, 35F20, 45K05, 47G20, 49L25, 35B10.

Key words and phrases. Particle system, periodic homogenization, Frenkel-Kontorova models, Hamilton-Jacobi equations, hull function.

(C)2012 American Mathematical Society 
Several results are related to our analysis. For instance in [5], homogenization results are obtained for monotone systems of Hamilton-Jacobi equations. Notice that they obtain a system at the limit, while we will obtain a single equation. Techniques from dynamical systems are also used to study systems of ODEs; see for instance [8, 18] and the references therein.

1.1. The classical overdamped Frenkel-Kontorova model. The classical Frenkel-Kontorova model describes a chain of classical particles evolving in a one dimensional space, coupled with their neighbours and subjected to a periodic potential. If $\tau$ denotes time and $U_{i}(\tau)$ denotes the position of the particle $i \in \mathbb{Z}$, one of the simplest FK models is given by the following dynamics:

$$
m_{0} \frac{d^{2} U_{i}}{d \tau^{2}}+\frac{d U_{i}}{d \tau}=U_{i+1}-2 U_{i}+U_{i-1}+\sin \left(2 \pi U_{i}\right)+L,
$$

where $m_{0}$ denotes the mass of the particle, $L$ is a constant driving force which can make the whole "train of particles" move and the term $\sin \left(2 \pi U_{i}\right)$ describes the force created by a periodic potential whose period is assumed to be 1 . Notice that in the previous equation, we set physical constants in front of the elastic and the exterior forces (friction and periodic potential). The goal of our work is to describe what is the macroscopic behaviour of the solution $U$ of (1.2) as the number of particles per length unit goes to infinity. As mentioned above, the particular case where $m_{0}=0$ is referred to as the fully overdamped one and has been studied in [10.

Next we would like to give the flavour of our main results. In order to do so, let us assume that, at initial time, particles satisfy

$$
\begin{aligned}
U_{i}(0) & =\varepsilon^{-1} u_{0}(i \varepsilon), \\
\frac{d U_{i}}{d \tau}(0) & =0
\end{aligned}
$$

for some $\varepsilon>0$ and some Lipschitz continuous function $u_{0}(x)$ which satisfies the following assumption:

\section{Initial gradient bounded from above and below:}

$$
0<1 / K_{0} \leq\left(u_{0}\right)_{x} \leq K_{0} \quad \text { on } \quad \mathbb{R}
$$

for some fixed $K_{0}>0$.

Such an assumption can be interpreted by saying that, at initial time, the number of particles per length unit lies in $\left(K_{0}^{-1} \varepsilon^{-1}, K_{0} \varepsilon^{-1}\right)$.

It is then natural to ask what is the macroscopic behaviour of the solution $U$ of (1.2) as $\varepsilon$ goes to zero, i.e. as the number of particles per length unit goes to infinity. To this end, we define the following function which describes the rescaled positions of the particles:

$$
\bar{u}^{\varepsilon}(t, x)=\varepsilon U_{\left\lfloor\varepsilon^{-1} x\right\rfloor}\left(\varepsilon^{-1} t\right),
$$

where $\lfloor\cdot\rfloor$ denotes the floor integer part. One of our main results states that the limiting dynamics as $\varepsilon$ goes to 0 of (1.2) is determined by a first order HamiltonJacobi equation of the form

$$
\begin{cases}u_{t}^{0}=\bar{F}\left(u_{x}^{0}\right) & \text { for } \quad(t, x) \in(0,+\infty) \times \mathbb{R}, \\ u^{0}(0, x)=u_{0}(x) & \text { for } \quad x \in \mathbb{R}\end{cases}
$$

where $\bar{F}$ is a continuous function to be determined. More precisely, we have the following homogenization result. 
Theorem 1.1 (Homogenization of the accelerated FK model). There exists a critical value $m_{0}^{c}$ such that for all $\left.\left.m_{0} \in\right] 0, m_{0}^{c}\right]$ and all $L \in \mathbb{R}$, there exists a continuous function $\bar{F}: \mathbb{R} \rightarrow \mathbb{R}$ such that, under assumption (1.3), the function $\bar{u}^{\varepsilon}$ converges locally uniformly towards the unique viscosity solution $u^{0}$ of (1.5).

Remark 1.2. The critical mass $m_{0}^{c}$ is made precise in assumption (A3) below.

1.2. Example of systems with $n$ types of particles. We now present the case of systems with $n$ types of particles. Let us start with the typical problem we have in mind. Let $n \in \mathbb{N} \backslash\{0\}$ be some integer and let us consider a sequence of real numbers $\left(\theta_{i}\right)_{i \in \mathbb{Z}}$ such that

$$
\theta_{i+n}=\theta_{i}>0 \quad \text { for all } \quad i \in \mathbb{Z} .
$$

It is then natural to consider the generalized FK model with $n$ different types of particles that stay ordered on the real line. Then, instead of satisfying (1.2), we can assume that $U_{i}$ satisfies for $\tau \in(0,+\infty)$ and $i \in \mathbb{Z}$,

$$
m_{0} \frac{d^{2} U_{i}}{d^{2} \tau}+\frac{d U_{i}}{d \tau}=\theta_{i+1}\left(U_{i+1}-U_{i}\right)-\theta_{i}\left(U_{i}-U_{i-1}\right)+\sin \left(2 \pi U_{i}\right)+L .
$$

Such a model is sketched in Figure 1. As we shall see, we can prove the same kind of homogenization results as Theorem 1.1.

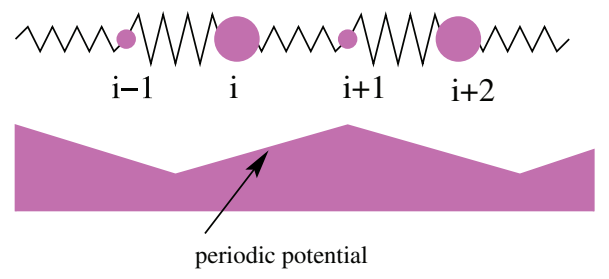

Figure 1. The FK model with $n=2$ type of particles (and of springs) and an interaction up to the $m=1$ neighbours.

As we mentioned before, it is crucial in our analysis to deal with monotone systems of ODEs. Inspired by the work of Baesens and MacKay 2 and of Hu, Qin and Zheng [12], we introduce for all $i \in \mathbb{Z}$ the following function:

$$
\Xi_{i}(\tau)=U_{i}(\tau)+2 m_{0} \frac{d U_{i}}{d \tau}(\tau) .
$$

Using this new function, the system of ODEs (1.6) can be rewritten in the following form: for $\tau \in(0,+\infty)$ and $i \in \mathbb{Z}$,

$$
\left\{\begin{array}{l}
\frac{d U_{i}}{d \tau}=\frac{1}{2 m_{0}}\left(\Xi_{i}-U_{i}\right), \\
\frac{d \Xi_{i}}{d \tau}=2 \theta_{i+1}\left(U_{i+1}-U_{i}\right)-2 \theta_{i}\left(U_{i}-U_{i-1}\right)+2 \sin \left(2 \pi U_{i}\right)+2 L+\frac{1}{2 m_{0}}\left(U_{i}-\Xi_{i}\right) .
\end{array}\right.
$$

We point out that, in comparison with [2, 12, our proof of the monotonicity of the system is simpler.

It is convenient to introduce the following notation:

$$
\alpha_{0}=\frac{1}{2 m_{0}} .
$$


Remark 1.3. It would also be possible to consider more generally $\Xi_{i}(\tau)=U_{i}(\tau)+$ $\frac{1}{\alpha} \frac{d U_{i}}{d \tau}(\tau)$ with $\frac{1}{\alpha}>m_{0}$. In order to simplify the presentation here, we choose $\alpha=1 /\left(2 m_{0}\right)$. Moreover, for the classical Frenkel-Kontorova model (1.2), the choice $\alpha=1 /\left(2 m_{0}\right)$ is optimal in the sense that the critical value $m_{0}^{c}$ for which the system is monotone is the best we can get.

1.3. General systems with $n$ types of particles. More generally, we would like to study the generalized Frenkel-Kontorova model (1.1) with $n$ types of particles. In order to do so, let us consider a general sequence of functions $v=\left(v_{j}(y)\right)_{j \in \mathbb{Z}}$ satisfying

$$
v_{j+n}(y)=v_{j}(y+1)
$$

For $m \in \mathbb{N}$, we set

$$
[v]_{j, m}(y)=\left(v_{j-m}(y), \ldots, v_{j+m}(y)\right) .
$$

We are going to study a function

$$
(u, \xi)=\left(\left(u_{j}(\tau, y)\right)_{j \in \mathbb{Z}},\left(\xi_{j}(\tau, y)\right)_{j \in \mathbb{Z}}\right)
$$

satisfying the following system of equations: for all $(\tau, y) \in(0,+\infty) \times \mathbb{R}$ and all $j \in \mathbb{Z}$

$$
\left\{\begin{array}{l}
\left\{\begin{array}{l}
\left(u_{j}\right)_{\tau}=\alpha_{0}\left(\xi_{j}-u_{j}\right), \\
\left(\xi_{j}\right)_{\tau}=2 F_{j}\left(\tau,[u(\tau, \cdot)]_{j, m}\right)+\alpha_{0}\left(u_{j}-\xi_{j}\right),
\end{array}\right. \\
\left\{\begin{array}{l}
u_{j+n}(\tau, y)=u_{j}(\tau, y+1) \\
\xi_{j+n}(\tau, y)=\xi_{j}(\tau, y+1)
\end{array}\right.
\end{array}\right.
$$

This system is referred to as the generalized Frenkel-Kontorova (FK for short) model. It is satisfied in the viscosity sense (see Definition 2.11). Moreover, we will consider viscosity solutions which are possibly discontinuous.

Let us now make precise the assumptions on the functions $F_{j}: \mathbb{R} \times \mathbb{R}^{2 m+1} \rightarrow \mathbb{R}$ mapping $(\tau, V)$ to $F_{j}(\tau, V)$. It is convenient to write $V \in \mathbb{R}^{2 m+1}$ as $\left(V_{-m}, \ldots, V_{m}\right)$.

(A1) (Regularity):

$$
\left\{\begin{array}{l}
F_{j} \text { is continuous, } \\
F_{j} \text { is Lipschitz continuous in } V \text { uniformly in } \tau \text { and } j .
\end{array}\right.
$$

(A2) (Monotonicity in $\left.V_{i}, i \neq 0\right)$ :

$$
F_{j}\left(\tau, V_{-m}, \ldots, V_{m}\right) \text { is non-decreasing in } V_{i} \quad \text { for } i \neq 0 .
$$

(A3) (Monotonicity in $V_{0}$ ):

$$
\alpha_{0}+2 \frac{\partial F_{j}}{\partial V_{0}} \geq 0 \quad \text { for all } j \in \mathbb{Z} .
$$

Keeping in mind the notation we chose above $\left(\alpha_{0}=\left(2 m_{0}\right)^{-1}\right)$, this assumption can be interpreted as follows: the mass has to be small in comparison with the variations of the non-linearity, which means that the system is sufficiently overdamped. This assumption guarantees that $2 F_{j}(\tau, V)+\alpha_{0} V_{0}$ is non-decreasing in $V_{0}$ for all $j \in \mathbb{Z}$.

(A4) (Periodicity):

$$
\left\{\begin{array}{l}
F_{j}\left(\tau, V_{-m}+1, \ldots, V_{m}+1\right)=F_{j}\left(\tau, V_{-m}, \ldots, V_{m}\right) \\
F_{j}(\tau+1, V)=F_{j}(\tau, V)
\end{array}\right.
$$




\section{(A5) (Periodicity of the type of particles):}

$$
F_{j+n}=F_{j} \quad \text { for all } \quad j \in \mathbb{Z}
$$

When $n=1$, we explained in [10] that the system of ODEs can be embedded into a single partial differential equation (more precisely, in a single ordinary differential equation with a real parameter $x$ ). Here, taking into account the " $n$-periodicity" of the indices $j$, it can be embedded into $n$ coupled systems of equations.

The next assumption allows us to guarantee that the ordering property of the particles, i.e. $u_{j} \leq u_{j+1}$, is preserved for all time.

(A6) (Ordering): For all $\left(V_{-m}, \ldots, V_{m}, V_{m+1}\right) \in \mathbb{R}^{2 m+2}$ such that $V_{i+1} \geq V_{i}$ for all $|i| \leq m$, we have

$$
2 F_{j+1}\left(\tau, V_{-m+1}, \ldots, V_{m+1}\right)+\alpha_{0} V_{1} \geq 2 F_{j}\left(\tau, V_{-m}, \ldots, V_{m}\right)+\alpha_{0} V_{0} .
$$

Remark 1.4. If, for all $j \in\{1, \ldots, n-1\}$, we have $F_{j+1}=F_{j}$, then assumption (A6) is a direct consequence of assumptions (A2) and (A3). Notice also that for $n \geq 1$, condition (A6') of Subsection 2.1 does not allow us to take $\alpha_{i}=\frac{1}{2 m_{i}}$ with different $m_{i}$ 's. In particular, all the particles in our analysis have the same mass $m_{0}$.

Example 1. We see that assumptions (A1)-(A5) are in particular satisfied for the FK system (1.6) with $n$ types of particles $\left(\theta_{n+j}=\theta_{j}\right), m=1$ and $F_{j}\left(\tau, V_{-1}, V_{0}, V_{1}\right)$ $=\theta_{j+1}\left(V_{1}-V_{0}\right)-\theta_{j}\left(V_{0}-V_{-1}\right)+\sin \left(2 \pi V_{0}\right)+L$ for $\alpha_{0} \geq 2\left(\theta_{j}+\theta_{j+1}\right)+4 \pi$. To get (A6) we have to assume furthermore that $\alpha_{0} \geq 4 \theta_{j}+4 \pi$.

We next rescale the generalized FK model: we consider for $\varepsilon>0$

$$
\left\{\begin{array}{l}
u_{j}^{\varepsilon}(t, x)=\varepsilon u_{j}\left(\frac{t}{\varepsilon}, \frac{x}{\varepsilon}\right), \\
\xi_{j}^{\varepsilon}(t, x)=\varepsilon \xi_{j}\left(\frac{t}{\varepsilon}, \frac{x}{\varepsilon}\right) .
\end{array}\right.
$$

The function $\left(u^{\varepsilon}, \xi^{\varepsilon}\right)=\left(\left(u_{j}^{\varepsilon}(t, x)\right)_{j \in \mathbb{Z}},\left(\xi_{j}^{\varepsilon}(t, x)\right)_{j \in \mathbb{Z}}\right)$ satisfies the following problem: for all $j \in \mathbb{Z}, t>0, x \in \mathbb{R}$

$$
\left\{\begin{array}{l}
\left\{\begin{array}{l}
\left(u_{j}^{\varepsilon}\right)_{t}=\alpha_{0} \frac{\xi_{j}^{\varepsilon}-u_{j}^{\varepsilon}}{\varepsilon} \\
\left(\xi_{j}^{\varepsilon}\right)_{t}=2 F_{j}\left(\frac{t}{\varepsilon},\left[\frac{u^{\varepsilon}(t, \cdot)}{\varepsilon}\right]_{j, m}\right)+\alpha_{0} \frac{u_{j}^{\varepsilon}-\xi_{j}^{\varepsilon}}{\varepsilon}
\end{array}\right. \\
\left\{\begin{array}{l}
u_{j+n}^{\varepsilon}(t, x)=u_{j}^{\varepsilon}(t, x+\varepsilon) \\
\xi_{j+n}^{\varepsilon}(t, x)=\xi_{j}^{\varepsilon}(t, x+\varepsilon)
\end{array}\right.
\end{array}\right.
$$


We impose the following initial conditions:

$$
\left\{\begin{array}{l}
u_{j}^{\varepsilon}(0, x)=u_{0}\left(x+\frac{j \varepsilon}{n}\right) \\
\xi_{j}^{\varepsilon}(0, x)=\xi_{0}^{\varepsilon}\left(x+\frac{j \varepsilon}{n}\right)
\end{array}\right.
$$

Finally, we assume that $u_{0}$ and $\xi_{0}^{\varepsilon}$ satisfy

(A0) (Gradient bound from below): There exist $K_{0}>0$ and $M_{0}>0$ such that

$$
\begin{array}{r}
0<1 / K_{0} \leq\left(u_{0}\right)_{x} \leq K_{0} \quad \text { on } \quad \mathbb{R} \\
0<1 / K_{0} \leq\left(\xi_{0}^{\varepsilon}\right)_{x} \leq K_{0} \quad \text { on } \mathbb{R} \\
\left\|u_{0}-\xi_{0}^{\varepsilon}\right\|_{\infty} \leq M_{0} \varepsilon
\end{array}
$$

Then we have the following homogenization result.

Theorem 1.5 (Homogenization of systems with $n$ types of particles). Assume that $\left(F_{j}\right)_{j}$ satisfies (A1)-(A6), and assume that the initial data $u_{0}, \xi_{0}^{\varepsilon}$ satisfy (A0). Consider the solution $\left(\left(u_{j}^{\varepsilon}\right)_{j \in \mathbb{Z}},\left(\xi_{j}^{\varepsilon}\right)_{j \in \mathbb{Z}}\right)$ of (1.8)-(1.9). Then, there exists a continuous function $\bar{F}: \mathbb{R} \mapsto \mathbb{R}$ such that, for all integers $j \in \mathbb{Z}$, the functions $u_{j}^{\varepsilon}$ and $\xi_{j}^{\varepsilon}$ converge uniformly on compact sets of $(0,+\infty) \times \mathbb{R}$ to the unique viscosity solution $u^{0}$ of (1.5).

Remark 1.6. The reader may be surprised by the fact that we obtain, at the limit, only one equation to describe the evolution of the system. In fact, this essentially comes from assumption (A6) and the definition of $\xi_{j}^{\varepsilon}$. Indeed, it could be shown that assumption (A6) implies that the functions $u^{\varepsilon}$ and $\xi^{\varepsilon}$ are non-decreasing with respect to $j: u_{j+1}^{\varepsilon} \geq u_{j}^{\varepsilon}$ and $\xi_{j+1}^{\varepsilon} \geq \xi_{j}^{\varepsilon}$. Then, the system can be essentially sketched by only two equations (one for the evolution of $u$ and one for $\xi$ ). But by the "microscopic definition" of $\xi_{j}^{\varepsilon}$, we have $\xi_{j}^{\varepsilon}=u_{j}^{\varepsilon}+O(\varepsilon)$; hence only one equation is sufficient to describe the macroscopic evolution of all of the system.

Remark 1.7. The case $m_{0}=0$ corresponds to $\alpha_{0}=+\infty$. In this case, $u^{\varepsilon} \equiv \xi^{\varepsilon}$ in (1.8), and Theorem 1.5 still holds true.

We will explain in the next subsection how the non-linearity $\bar{F}$, known as the effective Hamiltonian, is determined. We will see that this has to do with the existence of solutions of (1.8), (1.9) of a specific form. They are constructed thanks to functions referred to as hull functions.

1.4. Hull functions. In this subsection, we introduce the notion of a hull function for (1.7). More precisely, we look for special functions $\left(\left(h_{j}(\tau, z)\right)_{j \in \mathbb{Z}},\left(g_{j}(\tau, z)\right)_{j \in \mathbb{Z}}\right)$ such that $\left(u_{j}(\tau, y), \xi_{j}(\tau, y)\right)=\left(h_{j}(\tau, p y+\lambda \tau), g_{j}(\tau, p y+\lambda \tau)\right)$ is a solution of (1.7) on $\Omega=(-\infty,+\infty) \times \mathbb{R}=\mathbb{R}^{2}$. Here is a precise definition.

Definition 1.8 (Hull function for systems of $n$ types of particles). Given $\left(F_{j}\right)_{j}$ satisfying (A1)-(A6), $p \in(0,+\infty)$ and a number $\lambda \in \mathbb{R}$, we say that a family of functions $\left(\left(h_{j}\right)_{j},\left(g_{j}\right)_{j}\right)$ is a hull function for (1.7) if it satisfies for all $(\tau, z) \in \mathbb{R}^{2}$, $j \in \mathbb{Z}$ 


$$
\begin{aligned}
& \left\{\begin{array}{l}
\left(h_{j}\right)_{\tau}+\lambda\left(h_{j}\right)_{z}=\alpha_{0}\left(g_{j}-h_{j}\right), \\
h_{j}(\tau+1, z)=h_{j}(\tau, z), \\
h_{j}(\tau, z+1)=h_{j}(\tau, z)+1, \\
h_{j+n}(\tau, z)=h_{j}(\tau, z+p), \\
h_{j+1}(\tau, z) \geq h_{j}(\tau, z), \\
\left(h_{j}\right)_{z}(\tau, z) \geq 0, \\
\exists C \text { s.t. }\left|h_{j}(\tau, z)-z\right| \leq C,
\end{array}\right. \\
& \left\{\begin{array}{l}
\left(g_{j}\right)_{\tau}+\lambda\left(g_{j}\right)_{z}=2 F_{j}\left(\tau,[h(\tau, \cdot)]_{j, m}(z)\right)+\alpha_{0}\left(h_{j}-g_{j}\right), \\
g_{j}(\tau+1, z)=g_{j}(\tau, z), \\
g_{j}(\tau, z+1)=g_{j}(\tau, z)+1, \\
g_{j+n}(\tau, z)=g_{j}(\tau, z+p), \\
g_{j+1}(\tau, z) \geq g_{j}(\tau, z), \\
\left(g_{j}\right)_{z}(\tau, z) \geq 0, \\
\exists C \text { s.t. }\left|g_{j}(\tau, z)-z\right| \leq C .
\end{array}\right.
\end{aligned}
$$

In the case where the functions $\left(F_{j}\right)_{j}$ do not depend on $\tau$, we also require that the hull function $\left(\left(h_{j}\right)_{j},\left(g_{j}\right)_{j}\right)$ be independent on $\tau$ and denote it by $\left(\left(h_{j}(z)\right)_{j},\left(g_{j}(z)\right)_{j}\right)$.

Remark 1.9. The last line of (1.10) implies in particular that $\varepsilon h_{j}\left(\tau, \frac{z}{\varepsilon}\right) \rightarrow z$ and $\varepsilon g_{j}\left(\tau, \frac{z}{\varepsilon}\right) \rightarrow z$ as $\varepsilon \rightarrow 0$.

Given $p>0$, the following theorem explains how the effective Hamiltonian $\bar{F}(p)$ is determined by an existence/non-existence result of hull functions as $\lambda \in \mathbb{R}$ varies.

Theorem 1.10 (Effective Hamiltonian and hull function). Given $\left(F_{j}\right)_{j}$ satisfying (A1)-(A6) and $p \in(0,+\infty)$, there exists a unique real number $\lambda$ for which there exists a hull function $\left(\left(h_{j}\right)_{j},\left(g_{j}\right)_{j}\right)$ (depending on p) satisfying (1.10). Moreover, the real number $\lambda=\bar{F}(p)$, seen as a function of $p$, is continuous in $(0,+\infty)$.

1.5. Qualitative properties of the effective Hamiltonian. We have moreover the following result.

Theorem 1.11 (Qualitative properties of $\bar{F})$. Let $\left(F_{j}\right)_{j}$ satisfy (A1)-(A6). For any constant $L \in \mathbb{R}$, let $\bar{F}(L, p)$ denote the effective Hamiltonian given in Theorem 1.10 for $p \in(0,+\infty)$, associated with $\left(F_{j}\right)_{j}$ replaced by $\left(L+F_{j}\right)_{j}$.

Then $(L, p) \mapsto \bar{F}(L, p)$ is continuous and we have the following properties:

(i) (Bound): we have

$$
|\bar{F}(L, p)-L| \leq C_{p}
$$

(ii) (Monotonicity in $L$ ):

$$
\bar{F}(L, p) \text { is non-decreasing in } L \text {. }
$$

1.6. Organization of the article. In Section 2, we give some useful results concerning viscosity solutions for systems. In Section 3, we prove the convergence result assuming the existence of hull functions. The construction of hull functions is given in Sections 4 and 5. Finally, Section 6 is devoted to the proof of the qualitative properties of the effective Hamiltonian. 
1.7. Notation. Given $r, R>0, t \in \mathbb{R}$ and $x \in \mathbb{R}, Q_{r, R}(t, x)$ denotes the following neighbourhood of $(t, x)$ :

$$
Q_{r, R}(t, x)=(t-r, t+r) \times(x-R, x+R) .
$$

For $V=\left(V_{1}, \ldots, V_{N}\right) \in \mathbb{R}^{N},|V|_{\infty}$ denotes $\max _{j}\left|V_{j}\right|$. Given a family of functions $\left(v_{j}(\cdot)\right)_{j \in \mathbb{Z}}$ and two integers $j, m \in \mathbb{Z},[v]_{j, m}$ denotes the function $\left(v_{j-m}(\cdot), \ldots\right.$, $\left.v_{j+m}(\cdot)\right)$.

\section{VisCOSITY SOLUTIONS}

This section is devoted to the definition of viscosity solutions for systems of equations such as (1.7), (1.8) and (1.10). In order to construct hull functions when proving Theorem 1.10, we will also need to consider a perturbation of (1.7) with linear plus bounded initial data. For all these reasons, we define a viscosity solution for a generic equation whose Hamiltonian $\left(G_{j}\right)_{j}$ satisfies proper assumptions.

Before making precise assumptions, definitions and crucial results we will need later (such as stability, comparison principle, existence), we refer the reader to the user's guide of Crandall, Ishii, Lions [7] and the book of Barles [3] for an introduction to viscosity solutions and [6, 21, 16, 17] and the references therein for results concerning viscosity solutions for systems of weakly coupled partial differential equations.

2.1. Main assumptions and definitions. As we mentioned before, we consider systems with general non-linearities $\left(G_{j}\right)_{j}$. Precisely, for $0<T \leq+\infty$, we consider the following Cauchy problem: for $j \in \mathbb{Z}, \tau>0$ and $y \in \mathbb{R}$,

(2.1)

$$
\left\{\begin{array}{l}
\left\{\begin{array}{l}
\left(u_{j}\right)_{\tau}=\alpha_{0}\left(\xi_{j}-u_{j}\right), \\
\left(\xi_{j}\right)_{\tau}=G_{j}\left(\tau,[u(\tau, \cdot)]_{j, m}, \xi_{j}, \inf _{y^{\prime} \in \mathbb{R}}\left(\xi_{j}\left(\tau, y^{\prime}\right)-p y^{\prime}\right)+p y-\xi_{j}(\tau, y),\left(\xi_{j}\right)_{y}\right),
\end{array}\right. \\
\left\{\begin{array}{l}
u_{j+n}(\tau, y)=u_{j}(\tau, y+1), \\
\xi_{j+n}(\tau, y)=\xi_{j}(\tau, y+1)
\end{array}\right.
\end{array}\right.
$$

submitted to the initial conditions

$$
\left\{\begin{array}{l}
u_{j}(0, y)=u_{0}\left(y+\frac{j}{n}\right):=u_{0, j}(y), \\
\xi_{j}(0, y)=\xi_{0}\left(y+\frac{j}{n}\right):=\xi_{0, j}(y) .
\end{array}\right.
$$

Example 2. The most important example we have in mind is the following one:

$$
G_{j}\left(\tau, V_{-m}, \ldots, V_{m}, r, a, q\right)=2 F_{j}(\tau, V)+\alpha_{0}\left(V_{0}-r\right)+\delta\left(a_{0}+a\right) q^{+}
$$

for some constants $\delta \geq 0, a_{0}, a, q \in \mathbb{R}$ and where $F_{j}$ appears in (1.7), (1.8), (1.10).

In view of (2.1), it is clear that in the case where $G_{j}$ effectively depends on the variable $a$, solutions must be such that the infimum of $\xi_{j}(\tau, y)-p \cdot y$ is finite for all time $\tau$. Hence, when $G_{j}$ does depend on $a$, we will only consider solutions $\xi_{j}$ satisfying for some $C_{0}(T)>0$ : for all $\tau \in[0, T)$ and all $y, y^{\prime} \in \mathbb{R}$

$$
\left|\xi_{j}\left(\tau, y+y^{\prime}\right)-\xi_{j}(\tau, y)-p y^{\prime}\right| \leq C_{0} .
$$

When $T=+\infty$, we may assume that (2.3) holds true for all time $T_{0}>0$ for a family of constants $C_{0}>0$. 
Since we have to solve a Cauchy problem, we have to assume that the initial datum satisfies the assumption

$\left(\mathrm{A} 0^{\prime}\right)$ (Initial condition):

$\left(u_{0}, \xi_{0}\right)$ satisfies (A0) (with $\varepsilon=1$ ); it also satisfies (2.3) if $G_{j}$ depends on $a$ for some $j$.

As far as the $\left(G_{j}\right)_{j}$ 's are concerned, we make the following assumptions:

(A1') (Regularity):

(i) $G_{j}$ is continuous.

(ii) For all $R>0$, there exists $L_{0}=L_{0}(R)>0$ such that for all $\tau, V, W, r, s$, $a, q_{1}, q_{2}, j$, with $a \in[-R, R]$, we have

$\left|G_{j}\left(\tau, V, r, a, q_{1}\right)-G_{j}\left(\tau, W, s, a, q_{2}\right)\right| \leq L_{0}|V-W|_{\infty}+L_{0}|r-s|+L_{0}\left|q_{1}-q_{2}\right|$.

(iii) There exists $L_{1}>0$ such that for all $V, a, b, \tau, r, q$,

$$
\left|G_{j}(\tau, V, r, a, q)-G_{j}(\tau, V, r, b, q)\right| \leq L_{1}|a-b||q| .
$$

(A2') (Monotonicity in $\left.V_{i}, i \neq 0\right)$ :

$G_{j}\left(\tau, V_{-m}, \ldots, V_{m}, r, a, q\right)$ is non-decreasing in $V_{i}$ for $i \neq 0$.

(A3') (Monotonicity in $a$ and $\left.V_{0}\right)$ :

$$
G_{j}\left(\tau, V_{-m}, \ldots, V_{m}, r, a, q\right) \text { is non-decreasing in } a \text { and in } V_{0} \text {. }
$$

(A4') (Periodicity): For all $(\tau, V, r, a, q) \in \mathbb{R} \times \mathbb{R}^{2 m+1} \times \mathbb{R} \times \mathbb{R} \times \mathbb{R}$ and $j \in$ $\{1, \ldots, n\}$,

$$
\left\{\begin{array}{l}
G_{j}\left(\tau, V_{-m}+1, \ldots, V_{m}+1, r+1, a, q\right)=G_{j}\left(\tau, V_{-m}, \ldots, V_{m}, r, a, q\right), \\
G_{j}(\tau+1, V, r, a, q)=G_{j}(\tau, V, r, a, q) .
\end{array}\right.
$$

(A5') (Periodicity of the type of particles):

$$
G_{j+n}=G_{j} \quad \text { for all } j \in \mathbb{Z} .
$$

(A6') (Ordering): For all $\left(V_{-m}, \ldots, V_{m}, V_{m+1}\right) \in \mathbb{R}^{2 m+2}$ such that $\forall i, V_{i+1} \geq V_{i}$, we have

$$
G_{j+1}\left(\tau, V_{-m+1}, \ldots, V_{m+1}, r, a, q\right) \geq G_{j}\left(\tau, V_{-m}, \ldots, V_{m}, r, a, q\right) .
$$

Finally, we recall the definition of the upper and lower semi-continuous envelopes, $u^{*}$ and $u_{*}$, of a locally bounded function $u$ :

$$
u^{*}(\tau, y)=\limsup _{(t, x) \rightarrow(\tau, y)} u(t, x) \quad \text { and } \quad u_{*}(\tau, y)=\liminf _{(t, x) \rightarrow(\tau, y)} u(t, x) .
$$

We can now define viscosity solutions for (2.1).

Definition 2.1 (Viscosity solutions). Let $T>0$ and $u_{0}: \mathbb{R} \rightarrow \mathbb{R}$ and $\xi_{0}: \mathbb{R} \rightarrow \mathbb{R}$ be such that $\left(\mathrm{A} 0\right.$ ') is satisfied. For all $j$, consider locally bounded functions $u_{j}$ : $\mathbb{R}^{+} \times \mathbb{R} \rightarrow \mathbb{R}$ and $\xi_{j}: \mathbb{R}^{+} \times \mathbb{R} \rightarrow \mathbb{R}$. $\Omega$ denotes $(0, T] \times \mathbb{R}$.

- The function $\left(\left(u_{j}\right)_{j},\left(\xi_{j}\right)_{j}\right)$ is a sub-solution (resp. a super-solution) of (2.1) on $\Omega$ if (2.3) holds true for $\xi_{j}$ in the case where $G_{j}$ depends on $a$, and

$\forall j, n, \forall(\tau, y), \quad u_{j+n}(\tau, y)=u_{j}(\tau, y+1), \quad \xi_{j+n}(\tau, y)=\xi_{j}(\tau, y+1)$, and for all $j \in\{1, \ldots, n\}, u_{j}$ and $\xi_{j}$ are upper semi-continuous (resp. lower semi-continuous), and for all $(\tau, y) \in \Omega$ and any test function $\phi \in C^{1}(\Omega)$ 
such that $u_{j}-\phi$ attains a local maximum (resp. a local minimum) at the point $(\tau, y)$, then we have

$$
\phi_{\tau}(\tau, y) \leq \alpha_{0}\left(\xi_{j}(\tau, y)-u_{j}(\tau, y)\right) \quad(\text { resp. } \geq),
$$

and for all $(\tau, y) \in \Omega$ and any test function $\phi \in C^{1}(\Omega)$ such that $\xi_{j}-\phi$ attains a local maximum (resp. a local minimum) at the point $(\tau, y)$, then we have

$$
\begin{gathered}
\phi_{\tau}(\tau, y) \leq G_{j}\left(\tau,[u(\tau, \cdot)]_{j, m}(y), \xi_{j}(\tau, y), \inf _{y^{\prime} \in \mathbb{R}}\left(\xi_{j}\left(\tau, y^{\prime}\right)-p y^{\prime}\right)+p y-\xi_{j}(\tau, u), \phi_{y}(\tau, y)\right) \\
(\text { resp. } \geq) .
\end{gathered}
$$

- The function $\left(\left(u_{j}\right)_{j},\left(\xi_{j}\right)_{j}\right)$ is a sub-solution (resp. super-solution) of (2.1), (2.2) if $\left(\left(u_{j}\right)_{j},\left(\xi_{j}\right)_{j}\right)$ is a sub-solution (resp. super-solution) on $\Omega$ and if it satisfies moreover for all $y \in \mathbb{R}, j \in\{1, \ldots, n\}$,

$$
\begin{array}{ll}
u_{j}(0, y) \leq u_{0}\left(y+\frac{j}{n}\right) & (\text { resp. } \geq), \\
\xi_{j}(0, y) \leq \xi_{0}\left(y+\frac{j}{n}\right) & (\text { resp. } \geq) .
\end{array}
$$

- A function $\left(\left(u_{j}\right)_{j},\left(\xi_{j}\right)_{j}\right)$ is a viscosity solution of (2.1) (resp. of (2.1), (2.2)) if $\left(\left(u_{j}^{*}\right)_{j},\left(\xi_{j}^{*}\right)_{j}\right)$ is a sub-solution and $\left(\left(\left(u_{j}\right)_{*}\right)_{j},\left(\left(\xi_{j}\right)_{*}\right)_{j}\right)$ is a super-solution of (2.1) (resp. of (2.1), (2.2)).

Sub- and super-solutions satisfy the following comparison principle, which is a key property of the equation.

Proposition 2.2 (Comparison principle). Assume (A0') and that $\left(G_{j}\right)_{j}$ satisfies (A1')-(A5'). Let $\left(u_{j}, \xi_{j}\right)$ (resp. $\left.\left(v_{j}, \zeta_{j}\right)\right)$ be a sub-solution (resp. a super-solution) of (2.1), (2.2) such that (2.3) holds true for $\xi_{j}$ and $\zeta_{j}$ in the case where $G_{j}$ depends on a. We also assume that there exists a constant $K>0$ such that for all $j \in\{1, \ldots, n\}$ and $(t, x) \in[0, T] \times \mathbb{R}$, we have

$$
u_{j}(t, x) \leq u_{0, j}(x)+K(1+t), \quad \xi_{j}(t, x) \leq \xi_{0, j}(x)+K(1+t)
$$

(resp. $\left.-v_{j}(t, x) \leq-u_{0, j}(x)+K(1+t), \quad-\zeta_{j}(t, x) \leq-\xi_{0, j}(x)+K(1+t)\right)$.

If

$$
u_{j}(0, x) \leq v_{j}(0, x) \quad \text { and } \quad \xi_{j}(0, x) \leq \zeta_{j}(0, x) \quad \text { for all } j \in \mathbb{Z}, x \in \mathbb{R},
$$

then

$$
u_{j}(t, x) \leq v_{j}(t, x) \quad \text { and } \quad \xi_{j}(t, x) \leq \zeta_{j}(t, x) \quad \text { for all } j \in \mathbb{Z},(t, x) \in[0, T] \times \mathbb{R} .
$$

Remark 2.3. Even if it was not specified in [10, the Lipschitz continuity in $q$ of $G_{j}$ is necessary to obtain a general comparison principle.

Proof of Proposition 2.2. In view of assumption (A1')(i) and using the change of unknown functions $\bar{u}_{j}(t, x)=e^{-\lambda t} u_{j}(t, x)$ and $\bar{\xi}_{j}(t, x)=e^{-\lambda t} \xi_{j}(t, x)$, we classically assume, without loss of generality, that for all $r \geq s$,

$$
G_{j}(\tau, V, r, a, q)-G_{j}(\tau, V, s, a, q) \leq-L^{\prime}(r-s)
$$

for $L^{\prime} \geq L_{0}>0$.

Next we define

$$
M=\sup _{(t, x) \in(0, T) \times \mathbb{R}} \max _{j \in\{1, \ldots, n\}} \max \left(u_{j}(t, x)-v_{j}(t, x), \xi_{j}(t, x)-\zeta_{j}(t, x)\right) .
$$


The proof proceeds in several steps.

Step 1. The test function.

We argue by contradiction by assuming that $M>0$. Classically, we duplicate the space variable by considering for $\varepsilon, \alpha$ and $\eta$ "small" positive parameters, the functions

$$
\begin{aligned}
& \varphi(t, x, y, j)=u_{j}(t, x)-v_{j}(t, y)-e^{A t} \frac{|x-y|^{2}}{2 \varepsilon}-\alpha|x|^{2}-\frac{\eta}{T-t} \\
& \phi(t, x, y, j)=\xi_{j}(t, x)-\zeta_{j}(t, y)-e^{A t} \frac{|x-y|^{2}}{2 \varepsilon}-\alpha|x|^{2}-\frac{\eta}{T-t},
\end{aligned}
$$

where $A$ is a positive constant which will be chosen later. We also consider

$$
\Psi(t, x, y, j)=\max (\varphi(t, x, y, j), \phi(t, x, y, j)) .
$$

Using inequalities (2.6) and assumption (A0'), we get

$$
u_{j}(t, x)-v_{j}(t, y) \leq u_{0, j}(x)-u_{0, j}(y)+2 K(1+T) \leq K_{0}|x-y|+2 K(1+T)
$$

and

We then deduce that

$$
\xi_{j}(t, x)-\zeta_{j}(t, y) \leq K_{0}|x-y|+2 K(1+T) .
$$

$$
\lim _{|x|,|y| \rightarrow \infty} \varphi(t, x, y, j)=\lim _{|x|,|y| \rightarrow \infty} \phi(t, x, y, j)=-\infty .
$$

Also using the fact that $\varphi$ and $\phi$ are u.s.c, we deduce that $\Psi$ reaches its maximum at some point $(\bar{t}, \bar{x}, \bar{y}, \bar{j})$.

Let us assume that $\Psi(\bar{t}, \bar{x}, \bar{y}, \bar{j})=\phi(\bar{t}, \bar{x}, \bar{y}, \bar{j})$ (the other case being similar and even simpler). Using the fact that $M>0$, we first remark that for $\alpha$ and $\eta$ small enough, we have

In particular,

$$
\Psi(\bar{t}, \bar{x}, \bar{y}, \bar{j})=: M_{\varepsilon, \alpha, \eta} \geq \frac{M}{2}>0 .
$$

$$
\xi_{\bar{j}}(\bar{t}, \bar{x})-\zeta_{\bar{j}}(\bar{t}, \bar{y})>0 .
$$

Step 2. Viscosity inequalities for $\bar{t}>0$.

By duplicating the time variable and passing to the limit [7, 3, we classically get that there are real numbers $a, b, \bar{p} \in \mathbb{R}$ such that

$$
a-b=\frac{\eta}{(T-\bar{t})^{2}}+A e^{A \bar{t}} \frac{|\bar{x}-\bar{y}|^{2}}{2 \varepsilon}, \quad \bar{p}=e^{A \bar{t}} \frac{\bar{x}-\bar{y}}{\varepsilon}
$$

and

$$
\begin{aligned}
a & \leq G_{\bar{j}}\left(\bar{t},[u(\bar{t}, \cdot)]_{\bar{j}, m}(\bar{x}), \xi_{\bar{j}}(\bar{t}, \bar{x}), \inf \left(\xi_{\bar{j}}\left(\bar{t}, y^{\prime}\right)-p y^{\prime}\right)+p \bar{x}-\xi_{\bar{j}}(\bar{t}, \bar{x}), \bar{p}+2 \alpha \bar{x}\right), \\
b & \geq G_{\bar{j}}\left(\bar{t},[v(\bar{t}, \cdot)]_{\bar{j}, m}(\bar{y}), \zeta_{\bar{j}}(\bar{t}, \bar{y}), \inf \left(\zeta_{\bar{j}}\left(\bar{t}, y^{\prime}\right)-p y^{\prime}\right)+p \bar{y}-\zeta_{\bar{j}}(\bar{t}, \bar{y}), \bar{p}\right) .
\end{aligned}
$$

Subtracting the two above inequalities, we get

$$
\begin{aligned}
\frac{\eta}{T^{2}}+A e^{A \bar{t}} \frac{|\bar{x}-\bar{y}|^{2}}{2 \varepsilon} \leq & G_{\bar{j}}\left(\bar{t},[u(\bar{t}, \cdot)]_{\bar{j}, m}(\bar{x}), \xi_{\bar{j}}(\bar{t}, \bar{x}), \inf \left(\xi_{\bar{j}}\left(\bar{t}, y^{\prime}\right)-p y^{\prime}\right)\right. \\
& \left.+p \bar{x}-\xi_{\bar{j}}(\bar{t}, \bar{x}), \bar{p}+2 \alpha \bar{x}\right) \\
& -G_{\bar{j}}\left(\bar{t},[v(\bar{t}, \cdot)]_{\bar{j}, m}(\bar{y}), \zeta_{\bar{j}}(\bar{t}, \bar{y}), \inf \left(\zeta_{\bar{j}}\left(\bar{t}, y^{\prime}\right)-p y^{\prime}\right)\right. \\
& \left.+p \bar{y}-\zeta_{\bar{j}}(\bar{t}, \bar{y}), \bar{p}\right) \\
=: & \Delta G_{j} .
\end{aligned}
$$


Step 3. Estimate on $u_{k}(\bar{t}, \bar{x})-v_{k}(\bar{t}, \bar{y})$.

If $k \in\{1, \ldots, n\}$, by the inequality $\varphi(\bar{t}, \bar{x}, \bar{y}, k) \leq \phi(\bar{t}, \bar{x}, \bar{y}, \bar{j})$, we directly get that

$$
u_{k}(\bar{t}, \bar{x})-v_{k}(\bar{t}, \bar{y}) \leq \xi_{\bar{j}}(\bar{t}, \bar{x})-\zeta_{\bar{j}}(\bar{t}, \bar{y}) .
$$

If $k \notin\{1, \ldots, n\}$, let us define $l_{k} \in \mathbb{Z}$ such that $k-l_{k} n=\tilde{k} \in\{1, \ldots, n\}$. By periodicity, we then have

$$
\begin{aligned}
u_{k}(\bar{t}, \bar{x})-v_{k}(\bar{t}, \bar{y}) & =u_{\tilde{k}+l_{k} n}(\bar{t}, \bar{x})-v_{\tilde{k}+l_{k} n}(\bar{t}, \bar{y}) \\
& =u_{\tilde{k}}\left(\bar{t}, \bar{x}+l_{k}\right)-v_{\tilde{k}}\left(\bar{t}, \bar{y}+l_{k}\right) \\
& \leq \xi_{\bar{j}}(\bar{t}, \bar{x})-\zeta_{\bar{j}}(\bar{t}, \bar{y})-\alpha\left(|\bar{x}|^{2}-\left|\bar{x}+l_{k}\right|^{2}\right)
\end{aligned}
$$

where we have used the inequality $\varphi\left(\bar{t}, \bar{x}+l_{k}, \bar{y}+l_{k}, \tilde{k}\right) \leq \phi(\bar{t}, \bar{x}, \bar{y}, \bar{j})$ to get the third line. Hence, for all $k \in \mathbb{Z}$ (and in particular for $k \in\{\bar{j}-m, \ldots, \bar{j}+m\}$ ), we finally deduce that

$$
u_{k}(\bar{t}, \bar{x})-v_{k}(\bar{t}, \bar{y}) \leq \xi_{\bar{j}}(\bar{t}, \bar{x})-\zeta_{\bar{j}}(\bar{t}, \bar{y})+\left.\alpha|| \bar{x}\right|^{2}-\left|\bar{x}+l_{k}\right|^{2} \mid .
$$

Step 4. Estimate of $\Delta G_{j}$ in (2.8).

By successively using (2.9) and (A1')(ii), we obtain

$$
\begin{aligned}
\Delta G_{j} & \leq G_{\bar{j}}\left(\bar{t},\left[v(\bar{t}, \cdot)+\xi_{\bar{j}}(\bar{t}, \bar{x})-\zeta_{\bar{j}}(\bar{t}, \bar{y})+\left.\alpha|| \bar{x}\right|^{2}-|\bar{x}+l \cdot|^{2} \mid\right]_{\bar{j}, m}(\bar{y}), \xi_{\bar{j}}(\bar{t}, \bar{x}),\right. \\
& \left.\inf \left(\xi_{\bar{j}}\left(\bar{t}, y^{\prime}\right)-p y^{\prime}\right)+p \bar{x}-\xi_{\bar{j}}(\bar{t}, \bar{x}), \bar{p}+2 \alpha \bar{x}\right) \\
& -G_{\bar{j}}\left(\bar{t},[v(\bar{t}, \cdot)]_{\bar{j}, m}(\bar{y}), \zeta_{\bar{j}}(\bar{t}, \bar{y}), \inf \left(\zeta_{\bar{j}}\left(\bar{t}, y^{\prime}\right)-p y^{\prime}\right)+p \bar{y}-\zeta_{\bar{j}}(\bar{t}, \bar{y}), \bar{p}\right) \\
& \leq L_{0}\left(\xi_{\bar{j}}(\bar{t}, \bar{x})-\zeta_{\bar{j}}(\bar{t}, \bar{y})\right)+\left.L_{0} \alpha \max _{k \in\{\bar{j}-m, \ldots, \bar{j}+m\}}|| \bar{x}\right|^{2}-\left|\bar{x}+l_{k}\right|^{2} \mid \\
& +G_{\bar{j}}\left(\bar{t},[v(\bar{t}, \cdot)]_{\bar{j}, m}(\bar{y}), \xi_{\bar{j}}(\bar{t}, \bar{x}), \inf \left(\xi_{\bar{j}}\left(\bar{t}, y^{\prime}\right)-p y^{\prime}\right)+p \bar{x}-\xi_{\bar{j}}(\bar{t}, \bar{x}), \bar{p}+2 \alpha \bar{x}\right) \\
& -G_{\bar{j}}\left(\bar{t},[v(\bar{t}, \cdot)]_{\bar{j}, m}(\bar{y}), \zeta_{\bar{j}}(\bar{t}, \bar{y}), \inf \left(\zeta_{\bar{j}}\left(\bar{t}, y^{\prime}\right)-p y^{\prime}\right)+p \bar{y}-\zeta_{\bar{j}}(\bar{t}, \bar{y}), \bar{p}\right) .
\end{aligned}
$$

Now successively using (2.7) and (A1')(iii), we get

$$
\begin{aligned}
\Delta G_{j} & \leq L_{0}\left(\xi_{\bar{j}}(\bar{t}, \bar{x})-\zeta_{\bar{j}}(\bar{t}, \bar{y})\right)+\left.L_{0} \alpha \max _{k \in\{\bar{j}-m, \ldots, \bar{j}+m\}}|| \bar{x}\right|^{2}-\left|\bar{x}+l_{k}\right|^{2} \mid \\
& -L^{\prime}\left(\xi_{\bar{j}}(\bar{t}, \bar{x})-\zeta_{\bar{j}}(\bar{t}, \bar{y})\right) \\
& +G_{\bar{j}}\left(\bar{t},[v(\bar{t}, \cdot)]_{\bar{j}, m}(\bar{y}), \zeta_{\bar{j}}(\bar{t}, \bar{y}), \inf \left(\xi_{\bar{j}}\left(\bar{t}, y^{\prime}\right)-p y^{\prime}\right)+p \bar{x}-\xi_{\bar{j}}(\bar{t}, \bar{x}), \bar{p}+2 \alpha \bar{x}\right) \\
& -G_{\bar{j}}\left(\bar{t},[v(\bar{t}, \cdot)]_{\bar{j}, m}(\bar{y}), \zeta_{\bar{j}}(\bar{t}, \bar{y}), \inf \left(\zeta_{\bar{j}}\left(\bar{t}, y^{\prime}\right)-p y^{\prime}\right)+p \bar{y}-\zeta_{\bar{j}}(\bar{t}, \bar{y}), \bar{p}\right) \\
& \leq L \alpha \max _{k \in\{\bar{j}-m, \ldots, \bar{j}+m\}}\left(2\left|l_{k} \bar{x}\right|+l_{k}^{2}\right) \\
& +L_{1}\left(\inf \left(\xi_{\bar{j}}\left(\bar{t}, y^{\prime}\right)-p y^{\prime}\right)+p \bar{x}-\xi_{\bar{j}}(\bar{t}, \bar{x})-\inf \left(\zeta_{\bar{j}}\left(\bar{t}, y^{\prime}\right)-p y^{\prime}\right)\right. \\
& \left.\quad-p \bar{y}+\zeta_{\bar{j}}(\bar{t}, \bar{y})\right)^{+}|\bar{p}| \\
& +G_{\bar{j}}\left(\bar{t},[v(\bar{t}, \cdot)]_{\bar{j}, m}(\bar{y}), \zeta_{\bar{j}}(\bar{t}, \bar{y}), \inf \left(\xi_{\bar{j}}\left(\bar{t}, y^{\prime}\right)-p y^{\prime}\right)+p \bar{y}-\xi_{\bar{j}}(\bar{t}, \bar{x}), \bar{p}+2 \alpha \bar{x}\right) \\
& -G_{\bar{j}}\left(\bar{t},[v(\bar{t}, \cdot)]_{\bar{j}, m}(\bar{y}), \zeta_{\bar{j}}(\bar{t}, \bar{y}), \inf \left(\xi_{\bar{j}}\left(\bar{t}, y^{\prime}\right)-p y^{\prime}\right)+p \bar{y}-\xi_{\bar{j}}(\bar{t}, \bar{x}), \bar{p}\right) .
\end{aligned}
$$


Using the fact that $\alpha|\bar{x}| \rightarrow 0$ as $\alpha \rightarrow 0$, we deduce that

$$
\begin{aligned}
L \alpha \max _{k} & \left(2\left|l_{k} \bar{x}\right|+l_{k}^{2}\right) \\
& +G_{\bar{j}}\left(\bar{t},[v(\bar{t}, \cdot)]_{\bar{j}, m}(\bar{y}), \zeta_{\bar{j}}(\bar{t}, \bar{y}), \inf \left(\xi_{\bar{j}}\left(\bar{t}, y^{\prime}\right)-p y^{\prime}\right)+p \bar{y}-\xi_{\bar{j}}(\bar{t}, \bar{x}), \bar{p}+2 \alpha \bar{x}\right) \\
& -G_{\bar{j}}\left(\bar{t},[v(\bar{t}, \cdot)]_{\bar{j}, m}(\bar{y}), \zeta_{\bar{j}}(\bar{t}, \bar{y}), \inf \left(\xi_{\bar{j}}\left(\bar{t}, y^{\prime}\right)-p y^{\prime}\right)+p \bar{y}-\xi_{\bar{j}}(\bar{t}, \bar{y}), \bar{p}\right) \\
= & o_{\alpha}(1),
\end{aligned}
$$

where we have used (2.3) to get a uniform bound $R>0$ for $\inf \left(\xi_{\bar{j}}\left(\bar{t}, y^{\prime}\right)-p y^{\prime}\right)+$ $p \bar{y}-\xi_{\bar{j}}(\bar{t}, \bar{y})$.

Step 5. Passing to the limit.

Using the fact that $\phi\left(\bar{t}, y^{\prime}, y^{\prime}, \bar{j}\right) \leq \phi(\bar{t}, \bar{x}, \bar{y}, \bar{j})$, we deduce that

$$
\xi_{\bar{j}}\left(\bar{t}, y^{\prime}\right)-\xi_{\bar{j}}(\bar{t}, \bar{x}) \leq \zeta_{\bar{j}}\left(\bar{t}, y^{\prime}\right)-\zeta_{\bar{j}}(\bar{t}, \bar{y})+\alpha\left|y^{\prime}\right|^{2} .
$$

Combining this with the previous step, we get

$$
\begin{aligned}
\frac{\eta}{T^{2}}+A e^{A \bar{t}} \frac{|\bar{x}-\bar{y}|^{2}}{2 \varepsilon} & \leq L_{1}\left(\inf \left(\zeta_{\bar{j}}\left(\bar{t}, y^{\prime}\right)-p y^{\prime}-\zeta_{\bar{j}}(\bar{t}, \bar{y})+\alpha\left|y^{\prime}\right|^{2}\right)\right. \\
& \left.-\inf \left(\zeta_{\bar{j}}\left(\bar{t}, y^{\prime}\right)-p y^{\prime}-\zeta_{\bar{j}}(\bar{t}, \bar{y})\right)\right)^{+}|\bar{p}|+p(\bar{x}-\bar{y})|\bar{p}|+o_{\alpha}(1) \\
& \leq L_{1}\left(\inf \left(\zeta_{\bar{j}}\left(\bar{t}, y^{\prime}\right)-p y^{\prime}+\alpha\left|y^{\prime}\right|^{2}\right)-\inf \left(\zeta_{\bar{j}}\left(\bar{t}, y^{\prime}\right)-p y^{\prime}\right)\right)^{+}|\bar{p}| \\
& +p e^{A \bar{t}} \frac{|\bar{x}-\bar{y}|^{2}}{\varepsilon}+o_{\alpha}(1) .
\end{aligned}
$$

Choosing $A=2 p$, we finally get

$$
\frac{\eta}{T^{2}} \leq o_{\alpha}(1)+\left(\inf \left(\zeta_{\bar{j}}\left(\bar{t}, y^{\prime}\right)-p y^{\prime}+\alpha\left|y^{\prime}\right|^{2}\right)-\inf \left(\zeta_{\bar{j}}\left(\bar{t}, y^{\prime}\right)-p y^{\prime}\right)\right)|\bar{p}| .
$$

Using the fact that for $\bar{p}=O(1)$ when $\alpha \rightarrow 0$ (in fact the $O(1)$ depends on $\varepsilon$ which is fixed) and using classical arguments about inf-convolution, we get that

$$
\left(\inf \left(\zeta_{\bar{j}}\left(\bar{t}, y^{\prime}\right)-p y^{\prime}+\alpha\left|y^{\prime}\right|^{2}\right)-\inf \left(\zeta_{\bar{j}}\left(\bar{t}, y^{\prime}\right)-p y^{\prime}\right)\right)|\bar{p}|=o_{\alpha}(1),
$$

and so

$$
\frac{\eta}{T^{2}} \leq o_{\alpha}(1)
$$

which is a contradiction for $\alpha$ small enough.

Step 6. Case $\bar{t}=0$.

We assume that there exists a sequence $\varepsilon_{n} \rightarrow 0$ such that $\bar{t}=0$. In this case, we have

$0<\frac{M}{2} \leq M_{\varepsilon_{n}, \alpha, \eta} \leq \xi_{0}(\bar{x})-\xi_{0}(\bar{y})-\frac{|\bar{x}-\bar{y}|^{2}}{2 \varepsilon_{n}}-\alpha|x|^{2} \leq \xi_{0}(\bar{x})-\xi_{0}(\bar{y}) \leq\left\|D \xi_{0}\right\|_{L^{\infty}}|\bar{x}-\bar{y}|$.

Using the fact that $|\bar{x}-\bar{y}| \rightarrow 0$ as $\varepsilon_{n} \rightarrow 0$ yields a contradiction.

Let us now give a comparison principle on bounded sets. To this end, for a given point $\left(\tau_{0}, y_{0}\right) \in(0, T) \times \mathbb{R}$ and for all $r, R>0$, let us set

$$
Q_{r, R}=\left(\tau_{0}-r, \tau_{0}+r\right) \times\left(y_{0}-R, y_{0}+R\right) .
$$


We then have the following result whose proof is similar to the one of Proposition 2.2

Proposition 2.4 (Comparison principle on bounded sets). Assume (A1')-(A5') and that $G_{j}(\tau, V, r, a, q)$ does not depend on the variable a for each $j$. Assume that $\left(\left(u_{j}\right)_{j},\left(\xi_{j}\right)_{j}\right)$ is a sub-solution (resp. $\left(\left(v_{j}\right)_{j},(\zeta)_{j}\right)$ a super-solution) of (2.1) on the open set $Q_{r, R} \subset(0, T) \times \mathbb{R}$. Also assume that for all $j \in\{1, \ldots, n\}$

$$
u_{j} \leq v_{j} \quad \text { and } \quad \xi_{j} \leq \zeta_{j} \quad \text { on }\left(\bar{Q}_{r, R+m} \backslash Q_{r, R}\right)
$$

Then $u_{j} \leq v_{j}$ and $\xi_{j} \leq \zeta_{j}$ on $Q_{r, R}$ for $j \in\{1, \ldots, n\}$.

We now turn to the existence issue. Classically, we need to construct barriers for (2.1). In view of $\left(\mathrm{A}^{\prime}\right)(\mathrm{ii})$ and $\left(\mathrm{A} 4^{\prime}\right)$, for $K_{0}$ given in (A0), the quantity

$$
\bar{G}=\sup _{\tau \in \mathbb{R},|q| \leq K_{0}, j \in\{1, \ldots, n\}}\left|G_{j}(\tau, 0,0,0, q)\right|
$$

is finite. Let us also denote $L_{2}:=L_{1} K_{0}$. Hence, for all $\tau, a, b, r \in \mathbb{R}, V \in \mathbb{R}^{2 m+1}$, $q \in\left[-K_{0}, K_{0}\right]$ and $j \in\{1, \ldots, n\}$,

$$
\left|G_{j}(\tau, V, r, a, q)-G_{j}(\tau, V, r, b, q)\right| \leq L_{2}|a-b| .
$$

Then we have the following lemma.

Lemma 2.5 (Existence of barriers). Assume (A0')-(A5'). There exists a constant $K_{1}>0$ such that

$$
\left(\left(u_{j}^{+}(\tau, y)\right)_{j},\left(\xi_{j}^{+}(\tau, y)\right)_{j}\right)=\left(\left(u_{0}\left(y+\frac{j}{n}\right)+K_{1} \tau\right)_{j},\left(\xi_{0}\left(y+\frac{j}{n}\right)+K_{1} \tau\right)_{j}\right)
$$

and

$$
\left(\left(u_{j}^{-}(\tau, y)\right)_{j},\left(\xi_{j}^{-}(\tau, y)\right)_{j}\right)=\left(\left(u_{0}\left(y+\frac{j}{n}\right)-K_{1} \tau\right)_{j},\left(\xi_{0}\left(y+\frac{j}{n}\right)-K_{1} \tau\right)_{j}\right)
$$

are respectively super- and sub-solutions of (2.1), (2.2) for all $T>0$. Moreover, we can choose

$$
K_{1}=\max \left(L_{2} C_{0}+L_{0}\left(2+K_{0} \frac{m}{n}+M_{0}\right)+\bar{G}, \alpha_{0} M_{0}\right),
$$

where $C_{0},\left(K_{0}, M_{0}\right)$ and $\bar{G}$ are respectively given in (2.3), (A0') and (2.12).

Proof. We prove that $\left(\left(u_{j}^{+}(\tau, y)\right)_{j},\left(\xi_{j}^{+}(\tau, y)\right)_{j}\right)$ is a super-solution of (2.1), (2.2). In view of (A0) with $\varepsilon=1$, we have for all $j \in\{1, \ldots, n\}$

$$
\alpha_{0}\left(\xi_{j}^{+}(\tau, y)-u_{j}^{+}(\tau, y)\right)=\alpha_{0}\left(u_{0}\left(y+\frac{j}{n}\right)-\xi_{0}\left(y+\frac{j}{n}\right)\right) \leq \alpha_{0} M_{0} \leq K_{1}
$$


and

$$
\begin{aligned}
G_{j}\left(\tau,\left[u^{+}(\tau, \cdot)\right]_{j, m}(y), \xi_{j}^{+}(\tau, y), \inf _{y^{\prime} \in \mathbb{R}}\left(\xi_{j}^{+}\left(\tau, y^{\prime}\right)-p y^{\prime}\right)+p y-\xi_{j}^{+}(\tau, y),\left(\xi_{j}^{+}\right)_{y}(\tau, y)\right) \\
=G_{j}\left(\tau,\left[u^{+}(\tau, \cdot)-\left\lfloor u_{j}^{+}(\tau, y)\right\rfloor\right]_{j, m}(y), \xi_{j}^{+}(\tau, y)-\left\lfloor u_{j}^{+}(\tau, y)\right\rfloor,\right. \\
\left.\quad \inf _{y^{\prime} \in \mathbb{R}}\left(\xi_{0}\left(y^{\prime}+\frac{j}{n}\right)-p y^{\prime}\right)+p y-\xi_{0}\left(y+\frac{j}{n}\right),\left(\xi_{0}\right)_{y}\left(y+\frac{j}{n}\right)\right) \\
\leq \quad L_{2} C_{0}+L_{0}+L_{0}+G_{j}\left(\tau,\left[u^{+}(\tau, \cdot)-u_{j}^{+}(\tau, y)\right]_{j, m}(y),\right. \\
\left.\quad \xi_{j}^{+}(\tau, y)-u_{j}^{+}(\tau, y), 0,\left(\xi_{0}\right)_{y}\left(y+\frac{j}{n}\right)\right) \\
\leq L_{2} C_{0}+L_{0}+L_{0}+L_{0} K_{0} \frac{m}{n}+L_{0} M_{0}+G_{j}\left(\tau, 0, \ldots, 0,0,0,\left(\xi_{0}\right)_{y}\left(y+\frac{j}{n}\right)\right) \\
\leq \quad L_{2} C_{0}+2 L_{0}+L_{0} K_{0} \frac{m}{n}+L_{0} M_{0}+\bar{G},
\end{aligned}
$$

where we have used the periodicity assumption (A4') for the second line, assumptions (A0') and (A1')(ii) for the third line, the fact that $\left|u_{0}\left(y+\frac{j+k}{n}\right)-u_{0}\left(y+\frac{j}{n}\right)\right| \leq$ $K_{0} \frac{m}{n}$ for $|k| \leq m$ and assumption (A0') for the fourth line and $\left|\left(\xi_{j}^{+}\right)_{y}\right| \leq K_{0}$ for the last line.

When $G_{j}(\tau, V, r, a, q)$ is independent on $a$, we can simply choose $L_{2}=0$. This ends the proof of the lemma.

By applying Perron's method together with the comparison principle, we immediately get from the existence of barriers the following result.

Theorem 2.6 (Existence and uniqueness for (2.1)). Assume (A0')-(A5'). Then there exists a unique solution $\left(\left(u_{j}\right)_{j},\left(\xi_{j}\right)_{j}\right)$ of (2.1), (2.2). Moreover, the functions $u_{j}, \xi_{j}$ are continuous for all $j$.

We now claim that particles are ordered.

Proposition 2.7 (Ordering of the particles). Assume (A0') and that the $\left(G_{j}\right)_{j}$ 's satisfy (A1')-(A6'). Let $\left(u_{j}, \xi_{j}\right)$ be a solution of (2.1)-(2.2) such that (2.3) holds true for $\xi_{j}$ if $G_{j}$ depends on a. Also assume that the $u_{j}$ 's are Lipschitz continuous in space and let $L_{u}$ denote a common Lipschitz constant. Then $u_{j}$ and $\xi_{j}$ are non-decreasing with respect to $j$.

Proof of Proposition 2.7. The idea of the proof is to define $\left(v_{j}, \zeta_{j}\right)=\left(u_{j+1}, \xi_{j+1}\right)$. In particular, we have

$$
\left(v_{j}(0, y), \zeta_{j}(0, y)\right) \geq\left(u_{j}(0, y), \xi_{j}(0, y)\right)
$$


Moreover, $\left(\left(v_{j}\right)_{j},\left(\zeta_{j}\right)_{j}\right)$ is a solution of

$$
\left\{\begin{array}{l}
\left\{\begin{array}{l}
\left(v_{j}\right)_{\tau}=\alpha_{0}\left(\zeta_{j}-v_{j}\right), \\
\left(\zeta_{j}\right)_{\tau}=G_{j+1}\left(\tau,[v(\tau, \cdot)]_{j, m}, \zeta_{j}, \inf _{y^{\prime} \in \mathbb{R}}\left(\zeta_{j}\left(\tau, y^{\prime}\right)-p y^{\prime}\right)+p y-\zeta_{j}(\tau, y),\left(\zeta_{j}\right)_{y}\right),
\end{array}\right. \\
\left\{\begin{array}{l}
v_{j+n}(\tau, y)=v_{j}(\tau, y+1), \\
\zeta_{j+n}(\tau, y)=\zeta_{j}(\tau, y+1),
\end{array}\right. \\
\left\{\begin{array}{l}
v_{j}(0, y)=u_{0}\left(y+\frac{j}{n}\right), \\
\zeta_{j}(0, y)=\xi_{0}\left(y+\frac{j}{n}\right) .
\end{array}\right.
\end{array}\right.
$$

Now the goal is to obtain $u_{j} \leq v_{j}$ and $\xi_{j} \leq \zeta_{j}$. The arguments are essentially the same as those used in the proof of the comparison principle. The main difference is that (2.8) is replaced with

$$
\begin{aligned}
\frac{\eta}{T^{2}}+ & A e^{A \bar{t}} \frac{|\bar{x}-\bar{y}|^{2}}{2 \varepsilon} \\
\leq & G_{\bar{j}}\left(\bar{t},[u(\bar{t}, \cdot)]_{\bar{j}, m}(\bar{x}), \xi_{\bar{j}}(\bar{t}, \bar{x}), \inf \left(\xi_{\bar{j}}\left(\bar{t}, y^{\prime}\right)-p y^{\prime}\right)+p \bar{x}-\xi_{\bar{j}}(\bar{t}, \bar{x}), \bar{p}+2 \alpha \bar{x}\right) \\
& -G_{\bar{j}+1}\left(\bar{t},[v(\bar{t}, \cdot)]_{\bar{j}, m}(\bar{y}), \zeta_{\bar{j}}(\bar{t}, \bar{y}), \inf \left(\zeta_{\bar{j}}\left(\bar{t}, y^{\prime}\right)-p y^{\prime}\right)+p \bar{y}-\zeta_{\bar{j}}(\bar{t}, \bar{y}), \bar{p}\right) \\
\leq & G_{\bar{j}}\left(\bar{t},[u(\bar{t}, \cdot)]_{\bar{j}, m}(\bar{y}), \xi_{\bar{j}}(\bar{t}, \bar{x}), \inf \left(\xi_{\bar{j}}\left(\bar{t}, y^{\prime}\right)-p y^{\prime}\right)+p \bar{x}-\xi_{\bar{j}}(\bar{t}, \bar{x}), \bar{p}+2 \alpha \bar{x}\right) \\
& -G_{\bar{j}+1}\left(\bar{t},[v(\bar{t}, \cdot)]_{\bar{j}, m}(\bar{y}), \zeta_{\bar{j}}(\bar{t}, \bar{y}), \inf \left(\zeta_{\bar{j}}\left(\bar{t}, y^{\prime}\right)-p y^{\prime}\right)+p \bar{y}-\zeta_{\bar{j}}(\bar{t}, \bar{y}), \bar{p}\right) \\
& +L_{0} L_{u}|\bar{x}-\bar{y}| \\
= & \bar{\Delta} G_{j},
\end{aligned}
$$

where we have used the Lipschitz continuity of $u$ and assumption (A1').

To obtain the desired contradiction, we have to estimate the right hand side of this inequality. First, using Step 3 of the proof of the comparison principle (with the same notation), we can define

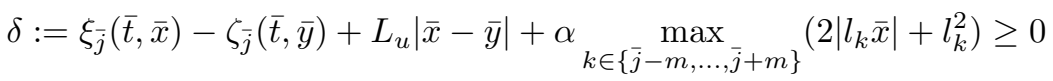

such that for $k \in\{\bar{j}-m, \ldots, \bar{j}+m\}$, we get from (2.9) the following estimate:

$$
u_{k}(\bar{t}, \bar{y})-v_{k}(\bar{t}, \bar{y}) \leq \delta .
$$

Using monotonicity assumptions (A2')-(A3') together with (A1'), we get

$$
\begin{aligned}
\bar{\Delta} G_{j} \leq & G_{\bar{j}}\left(\bar{t},[u(\bar{t}, \bar{y})+(\cdot-\bar{j}) \delta]_{\bar{j}, m}, \xi_{\bar{j}}(\bar{t}, \bar{x}), \inf \left(\xi_{\bar{j}}\left(\bar{t}, y^{\prime}\right)-p y^{\prime}\right)\right. \\
& \left.+p \bar{x}-\xi_{\bar{j}}(\bar{t}, \bar{x}), \bar{p}+2 \alpha \bar{x}\right) \\
& -G_{\bar{j}+1}\left(\bar{t},[v(\bar{t}, \bar{y})+(\cdot+1) \delta]_{\bar{j}, m}, \zeta_{\bar{j}}(\bar{t}, \bar{y}), \inf \left(\zeta_{\bar{j}}\left(\bar{t}, y^{\prime}\right)-p y^{\prime}\right)\right. \\
& \left.+p \bar{y}-\zeta_{\bar{j}}(\bar{t}, \bar{y}), \bar{p}\right) \\
& +L_{0}(2 m+1) \delta+L_{0} L_{u}|\bar{x}-\bar{y}| .
\end{aligned}
$$

Now we are going to use assumption (A6'). We remark first that we have for all $k \in\{-m, m-1\}$

$$
v_{\bar{j}+k}(\bar{t}, \bar{y})+(m+k+1) \delta=u_{\bar{j}+k+1}(\bar{t}, \bar{y})+(m+k+1) \delta,
$$

and for $k \in\{-m, \ldots, m\}$, (2.15) yields

$$
u_{\bar{j}+k+1}(\bar{t}, \bar{y})+(m+k+1) \delta \geq u_{\bar{j}+k}(\bar{t}, \bar{y})+(m+k) \delta .
$$


Thus (A6') implies that

$$
\begin{aligned}
& (2.16) \quad G_{\bar{j}}\left(\bar{t},[u(\bar{t}, \cdot)]_{\bar{j}, m}(\bar{y}), \xi_{\bar{j}}(\bar{t}, \bar{x}), \inf \left(\xi_{\bar{j}}\left(\bar{t}, y^{\prime}\right)-p y^{\prime}\right)+p \bar{x}-\xi_{\bar{j}}(\bar{t}, \bar{x}), \bar{p}+2 \alpha \bar{x}\right) \\
& \leq G_{\bar{j}+1}\left(\bar{t},[v(\bar{t}, \bar{y})+(\cdot+1) \delta]_{\bar{j}, m}, \xi_{\bar{j}}(\bar{t}, \bar{x}), \inf \left(\xi_{\bar{j}}\left(\bar{t}, y^{\prime}\right)-p y^{\prime}\right)+p \bar{x}-\xi_{\bar{j}}(\bar{t}, \bar{x}), \bar{p}+2 \alpha \bar{x}\right) .
\end{aligned}
$$

Hence

$$
\begin{aligned}
\bar{\Delta} G_{j} \leq & G_{\bar{j}+1}\left(\bar{t},[v(\bar{t}, \bar{y})+(\cdot+1) \delta]_{\bar{j}, m}, \xi_{\bar{j}}(\bar{t}, \bar{x}), \inf \left(\xi_{\bar{j}}\left(\bar{t}, y^{\prime}\right)-p y^{\prime}\right)\right. \\
& \left.+p \bar{x}-\xi_{\bar{j}}(\bar{t}, \bar{x}), \bar{p}+2 \alpha \bar{x}\right) \\
& -G_{\bar{j}+1}\left(\bar{t},[v(\bar{t}, \bar{y})+(\cdot+1) \delta]_{\bar{j}, m}, \zeta_{\bar{j}}(\bar{t}, \bar{y}), \inf \left(\zeta_{\bar{j}}\left(\bar{t}, y^{\prime}\right)-p y^{\prime}\right)\right. \\
& \left.+p \bar{y}-\zeta_{\bar{j}}(\bar{t}, \bar{y}), \bar{p}\right) \\
& +L_{0}(2 m+1)\left(\xi_{\bar{j}}(\bar{t}, \bar{x})-\zeta_{\bar{j}}(\bar{t}, \bar{y})\right)+2(m+1) L_{0} L_{u}|\bar{x}-\bar{y}| \\
& +L_{0}(2 m+1) \alpha \max _{k \in\{\bar{j}-m, \ldots, \bar{j}+m\}}\left(2\left|l_{k} \bar{x}\right|+l_{k}^{2}\right) .
\end{aligned}
$$

Now, to obtain the desired contradiction, it suffices to follow the computation from (2.10); in particular, choose $L^{\prime} \geq(2 m+1) L_{0}$ in (2.7). Then we obtain

$$
\frac{\eta}{T^{2}} \leq o_{\alpha}(1)+2(m+1) L_{0} L_{u}|\bar{x}-\bar{y}|
$$

which is absurd for $\alpha$ and $\varepsilon$ small enough (since $|\bar{x}-\bar{y}| \rightarrow 0$ as $\varepsilon \rightarrow 0$ ).

\section{Convergence}

This section is devoted to the proof of the main homogenization result (Theorem 1.5). The proof relies on the existence of hull functions (Theorem 1.10) and qualitative properties of the effective Hamiltonian (Theorem 1.11). As a matter of fact, we will use the existence of Lipschitz continuous sub- and super-hull functions (see Proposition 5.2). All these results are proved in the next sections.

We start with some preliminary results. Through a change of variables, the following result is a straightforward corollary of Lemma 2.5 and the comparison principle.

Lemma 3.1 (Barriers uniform in $\varepsilon$ ). Assume (A0)-(A5). Then there is a constant $C>0$ such that for all $\varepsilon>0$, the solution $\left(\left(u_{j}^{\varepsilon}\right)_{j},\left(\xi_{j}^{\varepsilon}\right)\right)$ of (1.8), (1.9) satisfies for all $t>0$ and $x \in \mathbb{R}$,

$$
\left|u_{j}^{\varepsilon}(t, x)-u_{0}\left(x+\frac{j \varepsilon}{n}\right)\right| \leq C t \quad \text { and } \quad\left|\xi_{j}^{\varepsilon}(t, x)-\xi_{0}^{\varepsilon}\left(x+\frac{j \varepsilon}{n}\right)\right| \leq C t .
$$

We also have the following preliminary lemma.

Lemma 3.2 ( $\varepsilon$-bounds on the gradient). Assume (A0)-(A5). Then the solution $\left(\left(u^{\varepsilon}\right)_{j},\left(\xi_{j}^{\varepsilon}\right)_{j}\right)$ of (1.8), (1.9) satisfies for all $t>0, x \in \mathbb{R}, z>0$ and $j \in \mathbb{Z}$,

$$
\varepsilon\left\lfloor\frac{z}{\varepsilon K_{0}}\right\rfloor \leq u_{j}^{\varepsilon}(t, x+z)-u_{j}^{\varepsilon}(t, x) \leq \varepsilon\left\lceil\frac{z K_{0}}{\varepsilon}\right\rceil
$$

and

$$
\varepsilon\left\lfloor\frac{z}{\varepsilon K_{0}}\right\rfloor \leq \xi_{j}^{\varepsilon}(t, x+z)-\xi_{j}^{\varepsilon}(t, x) \leq \varepsilon\left\lceil\frac{z K_{0}}{\varepsilon}\right\rceil .
$$

Remark 3.3. In particular, we obtain that functions $u_{j}^{\varepsilon}(t, x)$ and $\xi_{j}^{\varepsilon}(t, x)$ are nondecreasing in $x$. 
Proof of Lemma 3.2. We prove the bound from below (the proof is similar for the bound from above). We first remark that (A0) implies that the initial condition satisfies for all $j \in \mathbb{Z}$,

$u_{j}^{\varepsilon}(0, x+z)=u_{0}\left(x+z+\frac{j \varepsilon}{n}\right) \geq u_{0}\left(x+\frac{j \varepsilon}{n}\right)+z / K_{0} \geq u_{j}^{\varepsilon}(0, x)+k \varepsilon \quad$ with $\quad k=\left\lfloor\frac{z}{\varepsilon K_{0}}\right\rfloor$

and

$$
\xi_{j}^{\varepsilon}(0, x+z) \geq \xi_{j}^{\varepsilon}(0, x)+k \varepsilon .
$$

From (A4), we know that for $\varepsilon=1$, the equation is invariant by the addition of integers to solutions. After rescaling it, equation (1.8) is invariant by the addition of constants of the form $k \varepsilon, k \in \mathbb{Z}$. For this reason the solution of (1.8) associated with initial data $\left(\left(u_{j}^{\varepsilon}(0, x)+k \varepsilon\right)_{j},\left(\xi_{j}^{\varepsilon}(0, x)+k \varepsilon\right)_{j}\right)$ is $\left(\left(u_{j}^{\varepsilon}+k \varepsilon\right)_{j},\left(\xi_{j}^{\varepsilon}+k \varepsilon\right)_{j}\right)$. Similarly, the equation is invariant by space translations. Therefore the solution with initial data $\left(\left(u_{j}^{\varepsilon}(0, x+z)\right)_{j},\left(\xi_{j}^{\varepsilon}(0, x+z)\right)_{j}\right)$ is $\left(\left(u_{j}^{\varepsilon}(t, x+z)\right)_{j},\left(\xi_{j}^{\varepsilon}(t, x+z)\right)_{j}\right)$. Finally, from (3.2) and the comparison principle (Proposition 2.2), we get

$$
u_{j}^{\varepsilon}(t, x+z) \geq u_{j}^{\varepsilon}(t, x)+k \varepsilon \quad \text { and } \quad \xi_{j}^{\varepsilon}(t, x+z) \geq \xi_{j}^{\varepsilon}(t, x)+k \varepsilon
$$

which proves the bound from below. This ends the proof of the lemma.

We now turn to the proof of Theorem 1.5.

Proof of Theorem 1.5. We only have to prove the result for all $j \in\{1, \ldots, n\}$. Indeed, using the fact that $u_{j+n}^{\varepsilon}(t, x)=u_{j}^{\varepsilon}(t, x+\varepsilon)$ and $\xi_{j+n}^{\varepsilon}(t, x)=\xi_{j}^{\varepsilon}(t, x+\varepsilon)$, we will get the complete result.

For all $j \in\{1, \ldots, n\}$, we introduce the following half-relaxed limits:

$$
\begin{aligned}
& \bar{u}_{j}=\limsup _{\varepsilon \rightarrow 0}^{*} u_{j}^{\varepsilon}, \quad \bar{\xi}_{j}=\limsup _{\varepsilon \rightarrow 0}^{*} \xi_{j}^{\varepsilon}, \\
& \underline{u}_{j}=\liminf _{\varepsilon \rightarrow 0} u_{j}^{\varepsilon}, \quad \underline{\xi}_{j}=\liminf _{\varepsilon \rightarrow 0} \xi_{j}^{\varepsilon} .
\end{aligned}
$$

These functions are well defined thanks to Lemma 3.1. We then define

$$
\bar{v}=\max _{j \in\{1, \ldots, n\}} \max \left(\bar{u}_{j}, \bar{\xi}_{j}\right), \quad \underline{v}=\min _{j \in\{1, \ldots, n\}} \min \left(\underline{u}_{j}, \underline{\xi}_{j}\right) .
$$

We get from Lemmas 3.1 and 3.2 that both functions $w=\bar{v}, \underline{v}$ satisfy for all $t>0$, $x, x^{\prime} \in \mathbb{R}, x \leq x^{\prime}$ (recall that $\xi_{0}^{\varepsilon} \rightarrow u_{0}$ as $\varepsilon \rightarrow 0$ ),

$$
\begin{aligned}
\left|w(t, x)-u_{0}(x)\right| & \leq C t \\
K_{0}^{-1}\left|x-x^{\prime}\right| & \leq w(t, x)-w\left(t, x^{\prime}\right) \leq K_{0}\left|x-x^{\prime}\right| .
\end{aligned}
$$

We are going to prove that $\bar{v}$ is a sub-solution of (1.5). Similarly, we can prove that $\underline{v}$ is a super-solution of the same equation. Therefore, from the comparison principle for (1.5), we get that $u^{0} \leq \underline{v} \leq \bar{v} \leq u^{0}$. Then $\bar{v}=\underline{v}=u^{0}$, which shows the expected convergence of the full sequence $u_{j}^{\varepsilon}$ and $\xi_{j}^{\varepsilon}$ towards $u^{0}$ for all $j \in\{1, \ldots, n\}$.

We now prove in several steps that $\bar{v}$ is a sub-solution of (1.5). We classically argue by contradiction: we assume that there exists $(\bar{t}, \bar{x}) \in(0,+\infty) \times \mathbb{R}$ and a test function $\phi \in C^{1}$ such that

$$
\left\{\begin{array}{lll}
\bar{v}(\bar{t}, \bar{x})=\phi(\bar{t}, \bar{x}), & \\
\bar{v} \leq \phi & \text { on } \quad Q_{r, 2 r}(\bar{t}, \bar{x}), & \text { with } r>0 \\
\bar{v} \leq \phi-2 \eta & \text { on } \bar{Q}_{r, 2 r}(\bar{t}, \bar{x}) \backslash Q_{r, r}(\bar{t}, \bar{x}), & \text { with } \eta>0 \\
\phi_{t}(\bar{t}, \bar{x})=\bar{F}\left(\phi_{x}(\bar{t}, \bar{x})\right)+\theta, & \text { with } \theta>0
\end{array}\right.
$$


Let $p$ denote $\phi_{x}(\bar{t}, \bar{x})$. From (3.3), we get

$$
0<1 / K_{0} \leq p \leq K_{0}
$$

Then by combining Theorems 1.10 and 1.11, we get the existence of a hull function $\left(\left(h_{i}\right)_{i},\left(g_{i}\right)_{i}\right)$ associated with $p$ such that

$$
\lambda=\bar{F}(p)+\frac{\theta}{2}=\bar{F}(\bar{L}, p) \quad \text { with } \quad \bar{L}>0 .
$$

Indeed, we know from these results that the effective Hamiltonian is non-decreasing in $L$, continuous and goes to $\pm \infty$ as $L \rightarrow \pm \infty$.

Here we now apply the perturbed test function method introduced by Evans [9] in terms of hull functions instead of correctors. Precisely, let us consider the following twisted perturbed test functions for $i \in\{1, \ldots, n\}$ :

$$
\phi_{i}^{\varepsilon}(t, x)=\varepsilon h_{i}\left(\frac{t}{\varepsilon}, \frac{\phi(t, x)}{\varepsilon}\right), \quad \psi_{i}^{\varepsilon}(t, x)=\varepsilon g_{i}\left(\frac{t}{\varepsilon}, \frac{\phi(t, x)}{\varepsilon}\right) .
$$

Here the test functions are twisted in the same way as in 14. We then define the family of perturbed test functions $\left(\phi_{i}^{\varepsilon}\right)_{i \in \mathbb{Z}},\left(\left(\psi_{i}^{\varepsilon}\right)_{i \in \mathbb{Z}}\right)$ by using the following relation:

$$
\phi_{i+k n}^{\varepsilon}(t, x)=\phi_{i}^{\varepsilon}(t, x+\varepsilon k), \quad \psi_{i+k n}^{\varepsilon}(t, x)=\psi_{i}^{\varepsilon}(t, x+\varepsilon k) .
$$

In order to get a contradiction, we first assume that the functions $h_{i}$ and $g_{i}$ are $C^{1}$ and continuous in $z$ uniformly in $\tau \in \mathbb{R}, i \in\{1, \ldots, n\}$. In view of the third line of (1.10), we see that this implies that $h_{i}$ and $g_{i}$ are uniformly continuous in $z$ (uniformly in $\tau \in \mathbb{R}, i \in\{1, \ldots, n\}$ ). For simplicity, and since we will construct approximate hull functions with such a (Lipschitz) regularity, we even assume that $h_{i}$ and $g_{i}$ are globally Lipschitz continuous in $z$ (uniformly in $\tau \in \mathbb{R}, i \in\{1, \ldots, n\}$ ). We will next see how to treat the general case.

Case 1. $h_{i}$ and $g_{i}$ are $C^{1}$ and globally Lipschitz continuous in $z$.

Step 1.1. $\left(\left(\phi_{i}^{\varepsilon}\right)_{i},\left(\psi_{i}^{\varepsilon}\right)_{i}\right)$ is a super-solution of (1.8) in a neighbourhood of $(\bar{t}, \bar{x})$. When $h_{i}$ and $g_{i}$ are $C^{1}$, it is sufficient to check directly the super-solution property of $\left(\phi_{i}^{\varepsilon}, \psi_{i}^{\varepsilon}\right)$ for $(t, x) \in Q_{r, r}(\bar{t}, \bar{x})$. We begin with the equation satisfied by $\phi_{i}^{\varepsilon}$. We have, with $\tau=t / \varepsilon$ and $z=\phi(t, x) / \varepsilon$,

$$
\begin{aligned}
\left(\phi_{i}^{\varepsilon}\right)_{t}(t, x) & =\left(h_{i}\right)_{\tau}(\tau, z)+\phi_{t}(t, x)\left(h_{i}\right)_{z}(\tau, z) \\
& =\left(\phi_{t}(t, x)-\lambda\right)\left(h_{i}\right)_{z}(\tau, z)+\alpha_{0}\left(g_{i}(\tau, z)-h_{i}(\tau, z)\right) \\
& =\left(\phi_{t}(t, x)-\phi_{t}(\bar{t}, \bar{x})+\frac{\theta}{2}\right)\left(h_{i}\right)_{z}(\tau, z)+\frac{\alpha_{0}}{\varepsilon}\left(\psi_{i}^{\varepsilon}(t, x)-\phi_{i}^{\varepsilon}(t, x)\right) \\
& \geq \frac{\alpha_{0}}{\varepsilon}\left(\psi_{i}^{\varepsilon}(t, x)-\phi_{i}^{\varepsilon}(t, x)\right)
\end{aligned}
$$

where we have used the equation satisfied by $h_{i}$ to get the second line and the nonnegativity of $h_{z}$, the fact that $\theta>0$ and the fact that $\phi$ is $C^{1}$ in order to obtain the last line on $Q_{r, r}(\bar{t}, \bar{x})$ for $r>0$ small enough. 
We now turn to the equation satisfied by $\psi_{i}$. With the same notation, we have

$$
\begin{aligned}
& \left(\psi_{i}^{\varepsilon}\right)_{t}(t, x)-2 F_{i}\left(\tau,\left[\frac{\phi^{\varepsilon}(t, \cdot)}{\varepsilon}\right]_{i, m}(x)\right)-\frac{\alpha_{0}}{\varepsilon}\left(\phi_{i}^{\varepsilon}-\psi_{i}^{\varepsilon}\right) \\
& =\left(g_{i}\right)_{\tau}(\tau, z)+\phi_{t}(t, x)\left(g_{i}\right)_{z}(\tau, z)-2 F_{i}\left(\tau,\left[\frac{\phi^{\varepsilon}(t, \cdot)}{\varepsilon}\right]_{i, m}(x)\right)-\alpha_{0}\left(h_{i}(\tau, z)-g_{i}(\tau, z)\right) \\
& =\left(\phi_{t}(t, x)-\lambda\right)\left(g_{i}\right)_{z}(\tau, z)+2 \bar{L}+2\left(F_{i}\left(\tau,[h(\tau, \cdot)]_{i, m}(z)\right)-F_{i}\left(\tau,\left[\frac{\phi^{\varepsilon}(t, \cdot)}{\varepsilon}\right]_{i, m}(x)\right)\right) \\
& \geq\left(\phi_{t}(t, x)-\lambda\right)\left(g_{i}\right)_{z}(\tau, z)+2 \bar{L}-2 L_{F}\left|[h(\tau, \cdot)]_{i, m}(z)-\left[\frac{\phi^{\varepsilon}(t, \cdot)}{\varepsilon}\right]_{i, m}(x)\right|_{\infty},
\end{aligned}
$$

where we have used that equation (1.10) is satisfied by $\left(g_{i}\right)_{i}$ to get the third line and (A1) to get the fourth one. Here, $L_{F}$ denotes the largest Lipschitz constants of the $F_{i}$ 's (for $i \in\{1, \ldots, n\}$ ) with respect to $V$.

Let us next estimate, for $i \in\{1, \ldots, n\}, j \in\{-m, \ldots, m\}$ and $\varepsilon>0$,

$$
\mathcal{I}_{i, j}=h_{i+j}(\tau, z)-\frac{\phi_{i+j}^{\varepsilon}(t, x)}{\varepsilon} .
$$

If $i+j \in\{1, \ldots, n\}$, then, by the definition of $\phi_{i+j}$, we have

$$
\mathcal{I}_{i, j}=h_{i+j}\left(\frac{t}{\varepsilon}, \frac{\phi(t, x)}{\varepsilon}\right)-\frac{\phi_{i+j}^{\varepsilon}(t, x)}{\varepsilon}=0
$$

If $i+j \notin\{1, \ldots, n\}$, let us define $l$ such that $1 \leq i+j-l n \leq n$. We then have

$$
\begin{aligned}
\mathcal{I}_{i, j} & =h_{i+j-\ln }(\tau, z+l p)-\frac{\phi_{i+j-l n}^{\varepsilon}(t, x+\varepsilon l)}{\varepsilon} \\
& =h_{i+j-\ln }\left(\frac{t}{\varepsilon}, \frac{\phi(t, x)}{\varepsilon}+l p\right)-h_{i+j-\ln }\left(\frac{t}{\varepsilon}, \frac{\phi(t, x+\varepsilon l)}{\varepsilon}\right) \\
& =h_{i+j-\ln }\left(\frac{t}{\varepsilon}, \frac{\phi(t, x)}{\varepsilon}+l p\right)-h_{i+j-\ln }\left(\frac{t}{\varepsilon}, \frac{\phi(t, x)}{\varepsilon}+l p+o_{r}(1)\right),
\end{aligned}
$$

where $o_{r}(1)$ only depends on the modulus of continuity of $\phi_{x}$ on $Q_{r, r}(\bar{t}, \bar{x})$ (for $\varepsilon$ small enough such that $\varepsilon l \leq r$ with $l$ uniformly bounded and then $(t, x+\varepsilon l) \in$ $\left.Q_{r, 2 r}(\bar{t}, \bar{x})\right)$. Hence, if $h_{i}$ are Lipschitz continuous with respect to $z$ uniformly in $\tau$ and $i$, we conclude that we can choose $\varepsilon$ small enough so that

$$
\bar{L}-L_{F}\left|[h(\tau, \cdot)]_{i, m}(z)-\left[\frac{\phi^{\varepsilon}(t, \cdot)}{\varepsilon}\right]_{i, m}(x)\right|_{\infty} \geq 0 .
$$


Combining (3.7) and (3.8), we obtain

$$
\begin{aligned}
\left(\psi_{i}^{\varepsilon}\right)_{t} & (t, x)-2 F_{i}\left(\tau,\left[\frac{\phi^{\varepsilon}(t, x)}{\varepsilon}\right]_{i, m}(x)\right)+\frac{\alpha_{0}}{\varepsilon}\left(\phi_{i}^{\varepsilon}-\psi_{i}^{\varepsilon}\right) \\
& \geq\left(\phi_{t}(t, x)-\lambda\right)\left(g_{i}\right)_{z}(\tau, z) \\
& \geq\left(\frac{\theta}{2}+\phi_{t}(t, x)-\phi_{t}(\bar{t}, \bar{x})\right)\left(g_{i}\right)_{z}(\tau, z) \\
& =\left(\frac{\theta}{2}+o_{r}(1)\right)\left(g_{i}\right)_{z}(\tau, z) \geq 0 .
\end{aligned}
$$

We used the non-negativity of $\left(g_{i}\right)_{z}$, the fact that $\theta>0$ and again the fact that $\phi$ is $C^{1}$ in order to obtain the result on $Q_{r, r}(\bar{t}, \bar{x})$ for $r>0$ small enough. Therefore, when the $h_{i}$ and $g_{i}$ are $C^{1}$ and Lipschitz continuous on $z$ uniformly in $\tau$ and $i$, $\left(\left(\phi_{i}^{\varepsilon}\right)_{i},\left(\psi_{i}^{\varepsilon}\right)_{i}\right)$ is a viscosity super-solution of (1.8) on $Q_{r, r}(\bar{t}, \bar{x})$.

Step 1.2. Getting the contradiction.

By construction (see Remark [1.9), we have $\phi_{i}^{\varepsilon} \rightarrow \phi$ and $\psi_{i}^{\varepsilon} \rightarrow \phi$ as $\varepsilon \rightarrow 0$ for all $i \in$ $\{1, \ldots, n\}$, and therefore from the fact that $\bar{u}_{j} \leq \bar{v} \leq \phi-2 \eta$ on $\bar{Q}_{r, 2 r}(\bar{t}, \bar{x}) \backslash Q_{r, r}(\bar{t}, \bar{x})$ (see (3.4)), we get for $\varepsilon$ small enough that

$$
u_{i}^{\varepsilon} \leq \phi_{i}^{\varepsilon}-\eta \leq \phi_{i}^{\varepsilon}-\varepsilon k_{\varepsilon} \quad \text { on } \quad \bar{Q}_{r, 2 r}(\bar{t}, \bar{x}) \backslash Q_{r, r}(\bar{t}, \bar{x})
$$

with the integer

$$
k_{\varepsilon}=\lfloor\eta / \varepsilon\rfloor .
$$

In the same way, we have

$$
\xi_{i}^{\varepsilon} \leq \psi_{i}^{\varepsilon}-\eta \leq \psi_{i}^{\varepsilon}-\varepsilon k_{\varepsilon} \quad \text { on } \quad \bar{Q}_{r, 2 r}(\bar{t}, \bar{x}) \backslash Q_{r, r}(\bar{t}, \bar{x}) .
$$

Therefore, for $m \varepsilon \leq r$, we can apply the comparison principle on bounded sets to get

$$
u_{i}^{\varepsilon} \leq \phi_{i}^{\varepsilon}-\varepsilon k_{\varepsilon}, \quad \xi_{i}^{\varepsilon} \leq \psi_{i}^{\varepsilon}-\varepsilon k_{\varepsilon} \quad \text { on } \quad Q_{r, r}(\bar{t}, \bar{x}) .
$$

Passing to the limit as $\varepsilon$ goes to zero, we get

$$
\bar{u}_{i} \leq \phi-\eta, \quad \bar{\xi}_{i} \leq \phi-\eta \quad \text { on } \quad Q_{r, r}(\bar{t}, \bar{x}),
$$

which implies that

$$
\bar{v} \leq \phi-\eta \quad \text { on } \quad Q_{r, r}(\bar{t}, \bar{x}) .
$$

This gives a contradiction with $\bar{v}(\bar{t}, \bar{x})=\phi(\bar{t}, \bar{x})$ in (3.4). Therefore $\bar{v}$ is a subsolution of (1.5) on $(0,+\infty) \times \mathbb{R}$ and we get that $u_{j}^{\varepsilon}$ and $\xi_{j}^{\varepsilon}$ converge locally uniformly to $u^{0}$ for $j \in\{1, \ldots, n\}$. This ends the proof of the theorem.

Case 2. General case for $h$.

In the general case, we cannot check by a direct computation that $\left(\left(\phi_{i}^{\varepsilon}\right)_{i},\left(\psi_{i}^{\varepsilon}\right)_{i}\right)$ is a super-solution on $Q_{r, r}(\bar{t}, \bar{x})$. The difficulty is due to the fact that the $h_{i}$ and the $g_{i}$ may not be Lipschitz continuous in the variable $z$.

This kind of difficulty was overcome in [14 by using Lipschitz super-hull functions, i.e., functions satisfying (1.10), except that the function is only a supersolution of the equation appearing in the first line. Indeed, it is clear from the previous computations that it is enough for a conclusion. In [14, such regular super-hull functions (as a matter of fact, regular super-correctors) were built as 
exact solutions of an approximate Hamilton-Jacobi equation. Moreover, this Lipschitz continuous hull function is a super-solution for the exact Hamiltonian with a slightly larger $\lambda$.

Here we conclude by using a similar result, namely Proposition 5.2. Notice that in Proposition $5.2 h_{i}$ and $g_{i}$ are only Lipschitz continuous and not $C^{1}$. This is not a restriction, because the result of Step 1.1 can be checked in the viscosity sense using a test function (see 9 for further details). Comparing with [14, notice that we do not have to introduce an additional dimension because here $p>0$ (see (3.5)). This ends the proof of the theorem.

\section{ERgodicity AND CONSTRUCTION OF HULL FUNCTIONS}

In this section, we first study the ergodicity of equation (2.1) by studying the associated Cauchy problem (Subsection 4.1). We then construct hull functions (Subsection 4.2).

4.1. Ergodicity. In this subsection, we study the Cauchy problem associated with (2.1) by

$$
G_{j}(\tau, V, r, a, q)=G_{j}^{\delta}(\tau, V, r, a, q)=2 F_{j}(\tau, V)+\alpha_{0}\left(V_{0}-r\right)+\delta\left(a_{0}+a\right) q^{+},
$$

with $\delta \geq 0, a_{0} \in \mathbb{R}$ and with initial data $y \mapsto p y$. We prove that there exists a real number $\lambda$ (called the "slope in time" or "rotation number") such that the solution $\left(u_{j}, \xi_{j}\right)$ stays at a finite distance of the linear function $\lambda \tau+p y$. We also estimate this distance and give qualitative properties of the solution.

We begin by a regularity result concerning the solution of (2.1).

Proposition 4.1 (Bound on the gradient). Assume (A1)-(A5) and $p>0$. Let $\delta>0, a_{0} \in \mathbb{R}$ and $\left(u_{j}, \xi_{j}\right)_{j}$ be the solution of (2.1), (2.2) with $G_{j}=G_{j}^{\delta}$ defined by (4.1) and $u_{0}(y)=$ py. Assume that (2.3) holds true for $\xi_{j}$. Then $\left(u_{j}, \xi_{j}\right)_{j}$ satisfies

$$
0 \leq\left(u_{j}\right)_{y} \leq p+\frac{2 L_{F}}{\delta} \quad \text { and } \quad 0 \leq\left(\xi_{j}\right)_{y} \leq p+\frac{2 L_{F}}{\delta},
$$

where $L_{F}$ denotes the largest Lipschitz constant of the $F_{i}$ 's for $i=1, \ldots, n$.

Proof. We first show that $u_{j}$ and $\xi_{j}$ are non-decreasing with respect to $y$. Since equation (2.1) is invariant by translations in $y$ and using the fact that for all $b \geq 0$, we have

$$
u_{0}\left(y+b+\frac{j}{n}\right) \geq u_{0}\left(y+\frac{j}{n}\right)
$$

we deduce from the comparison principle that

$$
u_{j}(\tau, y+b) \geq u_{j}(\tau, y) \quad \text { and } \quad \xi_{j}(\tau, y+b) \geq \xi_{j}(\tau, y),
$$

which shows that $u_{j}$ and $\xi_{j}$ are non-decreasing in $y$.

We now explain how to get the Lipschitz estimate. We would like to prove that $\bar{M} \leq 0$, where

$$
\begin{aligned}
\bar{M}=\sup _{\tau \in(0, T), x, y \in \mathbb{R}, j \in\{1, \ldots, n\}} \max \{ & u_{j}(\tau, x)-u_{j}(\tau, y)-L|x-y|-\frac{\eta}{T-\tau}-\alpha|x|^{2}, \\
& \left.\xi_{j}(\tau, x)-\xi_{j}(\tau, y)-L|x-y|-\frac{\eta}{T-\tau}-\alpha|x|^{2}\right\}
\end{aligned}
$$


as soon as $L>p+\frac{2 L_{F}}{\delta}>0$ for any $\eta, \alpha>0$. We argue by contradiction by assuming that $\bar{M}>0$ for such an $L$. We next exhibit a contradiction. The supremum defining $\bar{M}$ is attained since $\xi_{j}$ satisfies (2.3) and $u_{j}$ can be explicitly computed.

Case 1. Assume that the supremum is attained for the function $u_{j}$ at $\tau \in[0, T)$, $j \in\{1, \ldots, n\}, x, y \in \mathbb{R}$. Since we have by assumption $\bar{M}>0$, this implies that $\tau>0, x \neq y$. Hence we can obtain the two following viscosity inequalities (by doubling the time variable and passing to the limit):

$$
\begin{aligned}
& a \leq \alpha_{0}\left(\xi_{j}(\tau, x)-u_{j}(\tau, x)\right), \\
& b \geq \alpha_{0}\left(\xi_{j}(\tau, y)-u_{j}(\tau, y)\right),
\end{aligned}
$$

with $a-b=\frac{\eta}{(T-\tau)^{2}}$. Subtracting these inequalities, we obtain

$$
\frac{\eta}{(T-\tau)^{2}} \leq \alpha_{0}\left(\left\{\xi_{j}(\tau, x)-\xi_{j}(\tau, y)\right\}-\left\{u_{j}(\tau, x)-u_{j}(\tau, y)\right\}\right) \leq 0 .
$$

We thus get $\eta \leq 0$, which is a contradiction in Case 1 .

Case 2. Assume next that the supremum is attained for the function $\xi_{j}$. By using the same notation and by arguing similarly, we obtain the following inequality:

$$
\begin{aligned}
\frac{\eta}{(T-\tau)^{2}} \leq & 2 F_{j}\left(\tau, u_{j-m}(\tau, x), \ldots, u_{j+m}(\tau, x)\right)-2 F_{j}\left(\tau, u_{j-m}(\tau, y), \ldots, u_{j+m}(\tau, y)\right) \\
& +\alpha_{0}\left(\left\{u_{j}(\tau, x)-u_{j}(\tau, y)\right\}-\left\{\xi_{j}(\tau, x)-\xi_{j}(\tau, y)\right\}\right) \\
& +\delta\left\{p(x-y)-\left(\xi_{j}(\tau, x)-\xi_{j}(\tau, y)\right)\right\} \operatorname{Lign}^{+}(x-y)+2 \alpha \delta\left(a_{0}+C_{0}\right)|x|,
\end{aligned}
$$

where $\operatorname{sign}^{+}$is the Heaviside function and where we have used (2.3). We now use

- the fact that the supremum is attained for the function $\xi_{j}$;

- the fact that $\xi_{j}(\tau, x)>\xi_{j}(\tau, y)$ implies that $x>y$ (remember that we already proved that $\xi_{j}$ is non-decreasing with respect to $y$ );

- assumption (A1); in the following, $L_{F}$ still denotes the largest Lipschitz constants of the $F_{j}$ 's with respect to $V$;

- the fact that $\alpha \delta\left(a_{0}+C_{0}\right)|x|=o_{\alpha}(1)$

in order to get the following inequality from the previous one:

$$
\begin{gathered}
\frac{\eta}{(T-\tau)^{2}} \leq 2 L_{F} \sup _{l \in\{-m, \ldots, m\}}\left|u_{j+l}(\tau, x)-u_{j+l}(\tau, y)\right|+\delta p L|x-y| \\
-L \delta\left(\xi_{j}(\tau, x)-\xi_{j}(\tau, y)\right)+o_{\alpha}(1) .
\end{gathered}
$$

Using the same computation as the one from the proof of Proposition 2.2. Step 3, we get

$$
\begin{aligned}
\sup _{l \in\{-m, \ldots, m\}}\left|u_{j+l}(\tau, x)-u_{j+l}(\tau, y)\right| & =\sup _{l \in\{-m, \ldots, m\}}\left(u_{j+l}(\tau, x)-u_{j+l}(\tau, y)\right) \\
& \leq \xi_{j}(\tau, x)-\xi_{j}(\tau, y)+C \alpha(1+|x|),
\end{aligned}
$$

where $C$ is a constant. Since $C \alpha(1+|x|)=o_{\alpha}(1)$ and $\bar{M}>0$, we finally deduce that

$\frac{\eta}{T^{2}} \leq 2 L_{F}\left(\xi_{j}(\tau, x)-\xi_{j}(\tau, y)\right)+\delta p\left(\xi_{j}(\tau, x)-\xi_{j}(\tau, y)\right)-L \delta\left(\xi_{j}(\tau, x)-\xi_{j}(\tau, y)\right)+o_{\alpha}(1)$.

For $\alpha$ small enough, it is now sufficient to once again use the fact that $\xi_{j}(\tau, x)>$ $\xi_{j}(\tau, y)$ and that $L>p+\frac{2 L_{F}}{\delta}$ in order to get the desired contradiction in Case 2. The proof is now complete. 
We now claim that particles are ordered.

Proposition 4.2 (Ordering of the particles). Assume (A0'), (A1)-(A6) and let $\delta \geq 0, a_{0} \in \mathbb{R}$ and $\left(u_{j}^{\delta}, \xi_{j}^{\delta}\right)_{j}$ be the solution of (2.1), (2.2) with $G_{j}=G_{j}^{\delta}$ defined by (4.1). Assume that (2.3) holds true for $\xi_{j}$ if $\delta>0$. Then $u_{j}^{\delta}$ and $\xi_{j}^{\delta}$ are nondecreasing with respect to $j$.

Proof. If $\delta>0$, the result is a straightforward consequence of Propositions 2.7 and 4.1. If $\delta=0$, the result is obtained by stability of viscosity solution i.e., $u_{j}^{\delta} \rightarrow u_{j}^{0}$ and $\xi_{j}^{\delta} \rightarrow \xi_{j}^{0}$ as $\left.\delta \rightarrow 0\right)$.

Proposition 4.3 (Ergodicity). Let $0 \leq \delta \leq 1$ and $a_{0} \in \mathbb{R}$. Assume (A0)-(A6) and let $\left(u_{j}, \xi_{j}\right)_{j}$ be a solution of (2.1), (2.2) with $G_{j}$ defined in (4.1) and with initial data $u_{0}(y)=\xi_{0}(y)=$ py with some $p>0$. Then there exists $\lambda \in \mathbb{R}$ such that for all $(\tau, y) \in[0,+\infty) \times \mathbb{R}, j \in\{1, \ldots, n\}$

$$
\left|u_{j}(\tau, y)-p y-\lambda \tau\right| \leq C_{3} \quad \text { and } \quad\left|\xi_{j}(\tau, y)-p y-\lambda \tau\right| \leq C_{3}
$$

and

$$
|\lambda| \leq C_{4}
$$

where

$$
\begin{aligned}
C_{3}=13+\frac{6 C_{4}}{\alpha_{0}}+7 p+2 K_{1}, & \\
C_{4}=\max \left(\alpha_{0} M_{0}, L_{F}(2+p(m+n))\right. & +\sup _{\tau}|F(\tau, 0, \ldots, 0)| \\
& \left.+\left(p / 2+L_{F}\right)\left(a_{0}+C_{0}\right)\right)
\end{aligned}
$$

(where $a_{0}$ is chosen equal to zero for $\delta=0$ ). Moreover, we have for all $\tau \geq 0$, $y, y^{\prime} \in \mathbb{R}, j \in\{1, \ldots, n\}$,

$$
\left\{\begin{array} { l } 
{ u _ { j } ( \tau , y + 1 / p ) = u _ { j } ( \tau , y ) + 1 , } \\
{ ( u _ { j } ) _ { y } ( \tau , y ) \geq 0 , } \\
{ | u _ { j } ( \tau , y + y ^ { \prime } ) - u _ { j } ( \tau , y ) - p y ^ { \prime } | \leq 1 , } \\
{ u _ { j + 1 } ( \tau , y ) \geq u _ { j } ( \tau , y ) , }
\end{array} \quad \left\{\begin{array}{l}
\xi_{j}(\tau, y+1 / p)=\xi_{j}(\tau, y)+1, \\
\left(\xi_{j}\right)_{y}(\tau, y) \geq 0 \\
\left|\xi_{j}\left(\tau, y+y^{\prime}\right)-\xi_{j}(\tau, y)-p y^{\prime}\right| \leq 1 \\
\xi_{j+1}(\tau, y) \geq \xi_{j}(\tau, y) .
\end{array}\right.\right.
$$

In order to prove Proposition 4.3 we will need the following classical lemma from ergodic theory (see for instance [19]).

Lemma 4.4. Consider $\Lambda: \mathbb{R}^{+} \rightarrow \mathbb{R}$ a continuous function which is sub-additive; that is to say, for all $t, s \geq 0$,

$$
\Lambda(t+s) \leq \Lambda(t)+\Lambda(s) .
$$

Then $\frac{\Lambda(t)}{t}$ has a limit $l$ as $t \rightarrow+\infty$ and

$$
l=\inf _{t>0} \frac{\Lambda(t)}{t} .
$$

We now turn to the proof of Proposition 4.3 ,

Proof of Proposition 4.3. We perform the proof in three steps. We first recall that the fact that $u_{j}$ and $\xi_{j}$ are non-decreasing in $y$ and $j$ follows from Propositions 4.1 and 4.2 . 
Step 1. Control of the space oscillations.

We are going to prove the following estimate.

Lemma 4.5. For all $\tau>0$, all $y, y^{\prime} \in \mathbb{R}$ and all $j \in\{1, \ldots, n\}$,

(4.7) $\left|u_{j}\left(\tau, y+y^{\prime}\right)-u_{j}(\tau, y)-p y^{\prime}\right| \leq 1 \quad$ and $\quad\left|\xi_{j}\left(\tau, y+y^{\prime}\right)-\xi_{j}(\tau, y)-p y^{\prime}\right| \leq 1$.

Proof. We have

$$
u_{j}(0, y+1 / p)=\xi_{j}(0, y+1 / p)=\xi_{j}(0, y)+1=u_{j}(0, y)+1 .
$$

Therefore from the comparison principle and from the integer periodicity of the Hamiltonian (see $\left(\mathrm{A} 3^{\prime}\right)$ ), we get that

$$
u_{j}(\tau, y+1 / p)=u_{j}(\tau, y)+1 \quad \text { and } \quad \xi_{j}(\tau, y+1 / p)=\xi_{j}(\tau, y)+1
$$

Since $u_{j}(\tau, y)$ is non-decreasing in $y$, we deduce that for all $b \in[0,1 / p]$,

$$
0 \leq u_{j}(\tau, b)-u_{j}(\tau, 0) \leq 1 .
$$

Now let $y \in \mathbb{R}$, and that we write $p y=k+a$ with $k \in \mathbb{Z}$ and $a \in[0,1)$. Then we have

$$
u_{j}(\tau, y)-u_{j}(\tau, 0)=k+u_{j}(\tau, a / p)-u_{j}(\tau, 0)
$$

which implies, for some $b \in[0,1 / p)$,

$$
u_{j}(\tau, y)-u_{j}(\tau, 0)-p y=-a+u_{j}(\tau, b)-u_{j}(\tau, 0) .
$$

Then for all $\tau>0$ and all $y \in \mathbb{R}$,

$$
\left|u_{j}(\tau, y)-u_{j}(\tau, 0)-p y\right| \leq 1 .
$$

In the same way, we get

$$
\left|\xi_{j}(\tau, y)-\xi_{j}(\tau, 0)-p y\right| \leq 1
$$

Finally, we obtain (4.7) by using the invariance by translations in $y$ of the problem.

Step 2. Estimate on $\left|u_{j}(\tau, y)-\xi_{j}(\tau, y)\right|$.

Lemma 4.6. For all $j \in\{1, \ldots, n\}$ and $0 \leq \delta \leq 1$,

$$
\left\|u_{j}-\xi_{j}\right\|_{L^{\infty}} \leq \frac{C_{4}}{\alpha_{0}}
$$

where $C_{4}$ is given by (4.5).

Proof. We recall that $\left(\left(u_{j}\right),\left(\xi_{j}\right)\right)$ is the solution of

$$
\left\{\begin{array}{l}
\left(u_{j}\right)_{\tau}=\alpha_{0}\left(\xi_{j}-u_{j}\right), \\
\left(\xi_{j}\right)_{\tau} \leq 2 F_{j}\left(\tau,[u(\tau, \cdot)]_{j, m}\right)+\alpha_{0}\left(u_{j}-\xi_{j}\right)+\delta\left(a_{0}+C_{0}\right)\left(\left(\xi_{j}\right)_{y}\right)^{+},
\end{array}\right.
$$

where we have used (2.3). Using Proposition 4.1 we deduce that (for $\delta \leq 1$ )

$$
\delta\left(a_{0}+C_{0}\right)\left(\left(\xi_{j}\right)_{y}\right)^{+} \leq\left(a_{0}+C_{0}\right)\left(p+2 L_{F}\right) .
$$

We now want to bound $F_{j}\left(\tau,[u(\tau, \cdot)]_{j, m}\right)$. We have

$$
\begin{aligned}
F_{j}\left(\tau,[u(\tau, \cdot)]_{j, m}(y)\right) & =F_{j}\left(\tau,\left[u(\tau, \cdot)-\left\lfloor u_{j}(\tau, y)\right\rfloor\right]_{j, m}(y)\right) \\
& \leq L_{F}+F_{j}\left(\tau,\left[u(\tau, \cdot)-u_{j}(\tau, y)\right]_{j, m}(y)\right) \\
& \leq L_{F}+L_{F} \sup _{k \in\{0, \ldots, m\}}\left(u_{j+k}(\tau, y)-u_{j}(\tau, y)\right)+\sup _{\tau} F(\tau, 0, \ldots, 0),
\end{aligned}
$$


where we have used the periodicity assumption (A4) for the first line, the Lipschitz regularity of $F$ for the second and third lines, and the fact that $u_{l}$ is non-decreasing with respect to $l$ for the third line. Moreover, for all $i \in\{1, \ldots, n\}, k \in\{0, \ldots m\}$, we have that

$$
\begin{aligned}
0 \leq u_{i+k}(\tau, y)-u_{i}(\tau, y) & =u_{i+k-\left\lceil\frac{k}{n}\right\rceil n}\left(\tau, y+\left\lceil\frac{k}{n}\right\rceil n\right)-u_{i}(\tau, y) \\
& \leq u_{i}\left(\tau, y+\left\lceil\frac{k}{n}\right\rceil n\right)-u_{i}(\tau, y) \\
& \leq 1+p\left\lceil\frac{k}{n}\right\rceil n \\
& \leq 1+p(m+n),
\end{aligned}
$$

where we have used the periodicity of $u_{i}$ for the first line, the monotonicity in $i$ of $u_{i}$ for the second one and the control of the oscillation (4.7) for the third one. We then deduce that

$$
F_{j}\left(\tau,\left[u_{j}(\tau, \cdot)\right]_{j, m}(y)\right) \leq L_{F}(2+p(m+n))+\sup _{\tau} F(\tau, 0, \ldots, 0) .
$$

Combining this inequality with (4.9) and (4.10), we deduce that

$$
\left\{\begin{array}{l}
\left(u_{j}\right)_{\tau}=\alpha_{0}\left(\xi_{j}-u_{j}\right) \\
\left(\xi_{j}\right)_{\tau} \leq 2 C_{4}+\alpha_{0}\left(u_{j}-\xi_{j}\right)
\end{array}\right.
$$

We now define for all $j \in \mathbb{Z}$ that $v_{j}=\xi_{j}-u_{j}$. Classical arguments from viscosity solution theory show that

$$
\left(v_{j}\right)_{\tau} \leq 2\left(C_{4}-\alpha_{0} v_{j}\right)
$$

We then deduce that

$$
v_{j} \leq \frac{C_{4}}{\alpha_{0}} .
$$

Using the same arguments with super-solution for $\xi_{j}$, we get the desired result.

Step 3. Control of the time oscillations.

We now explain how to control the time oscillations. The proof is inspired from [14. Let us introduce the following continuous functions defined for $T>0$ :

$$
\begin{aligned}
& \lambda_{+}^{u}(T)=\sup _{j \in\{1, \ldots, n\}} \sup _{\tau \geq 0} \frac{u_{j}(\tau+T, 0)-u_{j}(\tau, 0)}{T}, \\
& \lambda_{-}^{u}(T)=\inf _{j \in\{1, \ldots, n\}} \inf _{\tau \geq 0} \frac{u_{j}(\tau+T, 0)-u_{j}(\tau, 0)}{T},
\end{aligned}
$$

and

$$
\begin{aligned}
& \lambda_{+}^{\xi}(T)=\sup _{j \in\{1, \ldots, n\}} \sup _{\tau \geq 0} \frac{\xi_{j}(\tau+T, 0)-\xi_{j}(\tau, 0)}{T}, \\
& \lambda_{-}^{\xi}(T)=\inf _{j \in\{1, \ldots, n\}} \inf _{\tau \geq 0} \frac{\xi_{j}(\tau+T, 0)-\xi_{j}(\tau, 0)}{T},
\end{aligned}
$$

and

$$
\lambda_{+}(T)=\sup \left(\lambda_{+}^{u}(T), \lambda_{+}^{\xi}(T)\right) \quad \text { and } \quad \lambda_{-}(T)=\inf \left(\lambda_{-}^{u}(T), \lambda_{-}^{\xi}(T)\right) .
$$

In particular, these functions satisfy $-\infty \leq \lambda_{-}(T) \leq \lambda_{+}(T) \leq+\infty$. 
The goal is to prove that $\lambda_{+}(T)$ and $\lambda_{-}(T)$ have a common limit as $T \rightarrow \infty$. We would like to apply Lemma 4.4 .

In view of the definition of $\lambda_{+}^{u}$ and $\lambda_{+}^{\xi}$, we see that $T \mapsto T \lambda_{+}^{u}(T)$ and $T \mapsto$ $T \lambda_{+}^{\xi}(T)$ are sub-additive. Analogously, $T \mapsto-T \lambda_{-}^{u}(T)$ and $T \mapsto-T \lambda_{-}^{\xi}(T)$ are also sub-additive. Hence, if we can prove that these quantities $\lambda_{ \pm}^{u}(T), \lambda_{ \pm}^{\xi}(T)$ are finite, we will know that they converge. We will then have to prove that the limits of $\lambda_{+}$and $\lambda_{-}$are the same.

Step 3.1. First control on the time oscillations.

We first prove that $\lambda_{ \pm}$are finite.

Lemma 4.7. For all $T>0$,

$$
-K_{1}-\frac{C_{1}}{T} \leq \lambda_{-}(T) \leq \lambda_{+}(T) \leq K_{1}+\frac{C_{1}}{T},
$$

where $C_{1}=\frac{C_{4}}{\alpha_{0}}+3+2 p$ and $K_{1}$ is defined in (2.14).

Proof. Consider $j \in\{1, \ldots, n\}$. Using the control of the space oscillations (4.7), we get that

$$
u_{j}(\tau, y) \geq \Delta+p y-1 \quad \text { and } \quad \xi_{j}(\tau, y) \geq \Delta+p y-1,
$$

where

$$
\Delta=\inf _{j \in\{1, \ldots, n\}} \inf \left(u_{j}(\tau, 0), \xi_{j}(\tau, 0)\right) .
$$

Recalling (see Lemma 2.5) that $\lfloor\Delta-p\rfloor+p\left(y+\frac{j}{n}\right)-1-K_{1} t$ is a sub-solution and using the comparison principle on the time interval $[\tau, \tau+t)$, we deduce that

$u_{j}(\tau+t, y) \geq\lfloor\Delta-p\rfloor+p y+\frac{p j}{n}-1-K_{1} t \quad$ and $\quad \xi_{j}(\tau+t, y) \geq\lfloor\Delta-p\rfloor+p y+\frac{p j}{n}-1-K_{1} t$.

We now want to estimate $\Delta$ from below. Let us assume that the infimum in $\Delta$ is reached for the index $\bar{j} \in\{1, \ldots, n\}$. Then $\bar{j} \geq j-n$ since $j \in\{1, \ldots, n\}$. We then deduce that

$$
\begin{aligned}
p+\lfloor\Delta-p\rfloor & \geq \Delta-1 \\
& \geq u_{\bar{j}}(\tau, 0)-\frac{C_{4}}{\alpha_{0}}-1 \\
& \geq u_{j-n}(\tau, 0)-\frac{C_{4}}{\alpha_{0}}-1 \\
& \geq u_{j}(\tau,-1)-\frac{C_{4}}{\alpha_{0}}-1 \\
& \geq u_{j}(\tau, 0)-\frac{C_{4}}{\alpha_{0}}-2-p,
\end{aligned}
$$

where we have used (4.8) for the second line, the fact that $\left(u_{j}\right)_{j}$ is non-decreasing in $j$ for the third line, the periodicity of $u_{j}$ for the fourth line and (4.7) for the last one. In the same way, we get that

$$
p+\lfloor\Delta-p\rfloor \geq \xi_{j}(\tau, 0)-\frac{C_{4}}{\alpha_{0}}-2-p .
$$

Injecting this in (4.14), we get that

$$
u_{j}(\tau+t, y) \geq u_{j}(\tau, 0)-C_{1}+p y-K_{1} t
$$


and

$$
\xi_{j}(\tau+t, y) \geq \xi_{j}(\tau, 0)-C_{1}+p y-K_{1} t .
$$

In the same way, we also get

$$
u_{j}(\tau+t, y) \leq u_{j}(\tau, 0)+C_{1}+p y+K_{1} t
$$

and

$$
\xi_{j}(\tau+t, y) \leq \xi_{j}(\tau, 0)+C_{1}+p y+K_{1} t .
$$

Taking $y=0$, we finally get (4.13).

Step 3.2. Refined control on the time oscillations.

We now estimate $\lambda_{+}-\lambda_{-}$in order to prove that they have the same limit.

Lemma 4.8. For all $T>0$,

$$
\left|\lambda_{+}(T)-\lambda_{-}(T)\right| \leq \frac{C_{2}}{T},
$$

where $C_{2}=6+\frac{4 C_{4}}{\alpha_{0}}+3 p+2 C_{1}+2 K_{1}$.

Proof. By definition of $\lambda_{ \pm}(T)$, for all $\varepsilon>0$, there exists $\tau^{ \pm} \geq 0$ and $v^{ \pm} \in$ $\left\{u_{1}, \ldots, u_{n}, \xi_{1}, \ldots, \xi_{n}\right\}$ such that

$$
\left|\lambda_{ \pm}(T)-\frac{v^{ \pm}\left(\tau^{ \pm}+T, 0\right)-v^{ \pm}\left(\tau^{ \pm}, 0\right)}{T}\right| \leq \varepsilon .
$$

Consider $j \in\{1, \ldots, n\}$. We choose $\beta \in[0,1)$ such that $\tau^{+}{ }^{-} \tau^{-}-\beta=k \in \mathbb{Z}$, and we set

$$
\Delta_{j}^{u}=u_{j}\left(\tau^{+}, 0\right)-u_{j}\left(\tau^{-}+\beta, 0\right), \quad \Delta_{j}^{\xi}=\xi_{j}\left(\tau^{+}, 0\right)-\xi_{j}\left(\tau^{-}+\beta, 0\right)
$$

and

$$
\Delta=\sup _{j \in\{1, \ldots, n\}} \sup \left(\Delta_{j}^{u}, \Delta_{j}^{\xi}\right)
$$

Using (4.7), we get that

$$
u_{j}\left(\tau^{+}, y\right) \leq u_{j}\left(\tau^{-}+\beta, y\right)+2+\lceil\Delta\rceil \text { and } \xi_{j}\left(\tau^{+}, y\right) \leq \xi_{j}\left(\tau^{-}+\beta, y\right)+2+\lceil\Delta\rceil .
$$

Using the comparison principle, we then deduce that

$$
\begin{array}{r}
u_{j}\left(\tau^{+}+T, y\right) \leq u_{j}\left(\tau^{-}+\beta+T, y\right)+2+\lceil\Delta\rceil \\
\text { and } \xi_{j}\left(\tau^{+}+T, y\right) \leq \xi_{j}\left(\tau^{-}+\beta+T, y\right)+2+\lceil\Delta\rceil .
\end{array}
$$

We now want to estimate $\lceil\Delta\rceil$ from above. Let us assume that the maximum in $\Delta$ is reached for the index $\bar{j}$. We then have for all $j \in\{1, \ldots, n\}$,

$$
\begin{aligned}
\lceil\backslash\rceil & \leq u_{\bar{j}}\left(\tau^{+}, 0\right)-u_{\bar{j}}\left(\tau^{-}+\beta, 0\right)+\frac{2 C_{4}}{\alpha_{0}}+1 \\
& \leq u_{j+n}\left(\tau^{+}, 0\right)-u_{j-n}\left(\tau^{-}+\beta, 0\right)+\frac{2 C_{4}}{\alpha_{0}}+1 \\
& \leq u_{j}\left(\tau^{+}, 1\right)-u_{j}\left(\tau^{-}+\beta,-1\right)+\frac{2 C_{4}}{\alpha_{0}}+1 \\
& \leq u_{j}\left(\tau^{+}, 0\right)-u_{j}\left(\tau^{-}+\beta, 0\right)+\frac{2 C_{4}}{\alpha_{0}}+3+2 p,
\end{aligned}
$$


where we have used (4.8) for the first line, the fact that $\left(u_{j}\right)_{j}$ is non-decreasing in $j$ for the second line, the periodicity of $u_{j}$ for the third line and (4.7) for the last one. In the same way, we also get

$$
\lceil\Delta\rceil \leq \xi_{j}\left(\tau^{+}, 0\right)-\xi_{j}\left(\tau^{-}+\beta, 0\right)+\frac{2 C_{4}}{\alpha_{0}}+3+2 p .
$$

Injecting this in (4.17), we get

$$
u_{j}\left(\tau^{+}+T, y\right) \leq u_{j}\left(\tau^{-}+\beta+T, y\right)+5+\frac{2 C_{4}}{\alpha_{0}}+2 p+\Delta_{j}^{u}
$$

and

$$
\xi_{j}\left(\tau^{+}+T, y\right) \leq \xi_{j}\left(\tau^{-}+\beta+T, y\right)+5+\frac{2 C_{4}}{\alpha_{0}}+2 p+\Delta_{j}^{\xi} .
$$

Taking $y=0$ and using (4.15) (with $\tau=\tau^{-}$and $t=\beta$ ) and (4.16) (with $\tau=\tau^{-}+T$ and $t=\beta$ ), we get

$u_{j}\left(\tau^{+}+T, 0\right)-u_{j}\left(\tau^{+}, 0\right) \leq u_{j}\left(\tau^{-}+T, 0\right)-u_{j}\left(\tau^{-}, 0\right)+5+\frac{2 C_{4}}{\alpha_{0}}+2 p+2 C_{1}+2 K_{1}$.

In the same way, we get

$\xi_{j}\left(\tau^{+}+T, 0\right)-\xi_{j}\left(\tau^{+}, 0\right) \leq \xi_{j}\left(\tau^{-}+T, 0\right)-\xi_{j}\left(\tau^{-}, 0\right)+5+\frac{2 C_{4}}{\alpha_{0}}+2 p+2 C_{1}+2 K_{1}$.

Also using (4.8), (4.7) and the fact that $\left(u_{j}\right)_{j}$ and $\left(\xi_{j}\right)_{j}$ are non-decreasing in $j$, we finally get

$$
v^{+}\left(\tau^{+}+T, 0\right)-v^{+}\left(\tau^{+}, 0\right) \leq v^{-}\left(\tau^{-}+T, 0\right)-v^{-}\left(\tau^{-}, 0\right)+C_{2} .
$$

The comparison of $u_{j}$ and $\xi_{j}$ makes the additional constant $2 C_{4} / \alpha_{0}$ appear and the comparison between $u_{j}$ and $u_{k}$ (and similarly between $\xi_{j}$ and $\xi_{k}$ ) creates an additional constant $1+p$. Indeed, we have

$u_{j}(\tau, 0)-u_{k}(\tau, 0)=u_{j+n}(\tau, 1)-u_{k}(\tau, 0) \leq u_{j+n}(\tau, 0)-u_{k}(\tau, 0)+1+p \leq 1+p$.

This explains the value of the new constant $C_{2}$.

This implies that

$$
T \lambda_{+}(T) \leq T \lambda_{-}(T)+2 \varepsilon+C_{2} .
$$

Since this is true for all $\varepsilon>0$, the proof of the lemma is complete.

Step 3.3. Conclusion.

We now can conclude that $\lim _{T \rightarrow+\infty} \lambda_{ \pm}(T)$ is equal. If $\lambda$ denotes the common limit, we also have, by Lemma 4.4, that for every $T>0$,

$$
\lambda_{-}(T) \leq \lambda \leq \lambda_{+}(T) \text {. }
$$

Moreover, by Lemma 4.8, we have

and so

$$
\lambda_{+}(T) \leq \lambda_{-}(T)+\frac{C_{2}}{T},
$$

$$
\lambda_{-}(T) \leq \lambda \leq \lambda_{-}(T)+\frac{C_{2}}{T} .
$$

We finally deduce (using a similar argument for $\lambda_{+}$) that

$$
\left|\lambda_{ \pm}(T)-\lambda\right| \leq \frac{C_{2}}{T}
$$


Combining this estimate and (4.7), we get with $T=\tau$ that

$$
\left|u_{j}(\tau, y)-u_{j}(0,0)-p y-\lambda \tau\right| \leq C_{2}+1
$$

and

$$
\left|\xi_{j}(\tau, y)-\xi_{j}(0,0)-p y-\lambda \tau\right| \leq C_{2}+1 .
$$

This finally implies (4.3) with $C_{3}=C_{2}+1$.

4.2. Construction of hull functions for general Hamiltonians. In this subsection, we construct hull functions for a general Hamiltonian $G_{j}$. As we shall see, this is a straightforward consequence of the construction of time-space periodic solutions of (4.18); see Proposition 4.9 and Corollary 4.10 below. We will then prove that the time slope obtained in Proposition 4.3 is unique and that the map $p \mapsto \lambda$ is continuous; see Proposition 4.11 below.

Given $p>0$, we consider the equation in $\mathbb{R} \times \mathbb{R}$ :

$$
\left\{\begin{array}{l}
\left\{\begin{array}{l}
\left(u_{j}\right)_{\tau}=\alpha_{0}\left(\xi_{j}-u_{j}\right), \\
\left(\xi_{j}\right)_{\tau}=G_{j}\left(\tau,[u(\tau, \cdot)]_{j, m}, \xi_{j}, \inf _{y^{\prime} \in \mathbb{R}}\left(\xi_{j}\left(\tau, y^{\prime}\right)-p y^{\prime}\right)+p y-\xi_{j}(\tau, y),\left(\xi_{j}\right)_{y}\right),
\end{array}\right. \\
\left\{\begin{array}{l}
u_{j+n}(\tau, y)=u_{j}(\tau, y+1), \\
\xi_{j+n}(\tau, y)=\xi_{j}(\tau, y+1)
\end{array}\right.
\end{array}\right.
$$

where $G_{j}=G_{j}^{\delta}$ is given in (4.1) for $\delta \geq 0$. Then we have the following result.

Proposition 4.9 (Existence of time-space periodic solutions of (4.18)). Let $0 \leq$ $\delta \leq 1, a_{0} \in \mathbb{R}$ and $p>0$. Assume (A1)-(A6). Then there exist functions $\left(\left(u_{j}^{\infty}\right)_{j},\left(\xi_{j}^{\infty}\right)_{j}\right)$ solving (4.18) on $\mathbb{R} \times \mathbb{R}$ and a real number $\lambda \in \mathbb{R}$ satisfying for all $\tau, y \in \mathbb{R}, j \in\{1, \ldots, n\}$,

$$
\begin{aligned}
\left|u_{j}^{\infty}(\tau, y)-p y-\lambda \tau\right| & \leq 2\left\lceil C_{3}\right\rceil, \\
\left|\xi_{j}^{\infty}(\tau, y)-p y-\lambda \tau\right| & \leq 2\left\lceil C_{3}\right\rceil .
\end{aligned}
$$

Moreover, $\left(\left(u_{j}^{\infty}\right)_{j},\left(\xi_{j}^{\infty}\right)_{j}\right)$ satisfies for $j \in\{1, \ldots, n\}$,

$$
\left\{\begin{array} { l } 
{ u _ { j } ^ { \infty } ( \tau , y + 1 / p ) = u _ { j } ^ { \infty } ( \tau , y ) + 1 , } \\
{ u _ { j } ^ { \infty } ( \tau + 1 , y ) = u _ { j } ^ { \infty } ( \tau , y + \lambda / p ) , } \\
{ ( u _ { j } ^ { \infty } ) _ { y } ( \tau , y ) \geq 0 , } \\
{ u _ { j + 1 } ( \tau , y ) \geq u _ { j } ( \tau , y ) , }
\end{array} \quad \left\{\begin{array}{l}
\xi_{j}^{\infty}(\tau, y+1 / p)=\xi_{j}^{\infty}(\tau, y)+1 \\
\xi_{j}^{\infty}(\tau+1, y)=\xi_{j}^{\infty}(\tau, y+\lambda / p), \\
\left(\xi_{j}^{\infty}\right)_{y}(\tau, y) \geq 0, \\
\xi_{j+1}(\tau, y) \geq \xi_{j}(\tau, y)
\end{array}\right.\right.
$$

Eventually, when the Hamiltonians $G_{j}$ are independent on $\tau$, we can choose $u_{j}^{\infty}$ and $\xi_{j}^{\infty}$ independent on $\tau$.

By considering for all $\tau, z \in \mathbb{R}$

$$
\left\{\begin{array}{l}
h_{j}(\tau, z)=u_{j}^{\infty}(\tau,(z-\lambda \tau) / p) \quad \text { if } j \in\{1, \ldots, n\}, \\
h_{j+n}(\tau, z)=h_{j}(\tau, z+p) \text { otherwise }
\end{array}\right.
$$

and for all $\tau, z \in \mathbb{R}$

$$
\left\{\begin{array}{l}
g_{j}(\tau, z)=\xi_{j}^{\infty}(\tau,(z-\lambda \tau) / p) \text { if } j \in\{1, \ldots, n\} \\
g_{j+n}(\tau, z)=g_{j}(\tau, z+p) \text { otherwise }
\end{array}\right.
$$

we immediately get the following corollary. 
Corollary 4.10 (Existence of hull functions). Assume (A1)-(A6). There exists a hull function $\left(\left(h_{j}\right)_{j},\left(g_{j}\right)_{j}\right)$ in the sense of Definition 1.8 satisfying

$$
\left|h_{j}(\tau, z)-z\right| \leq 2\left\lceil C_{3}\right\rceil
$$

and

$$
\left|g_{j}(\tau, z)-z\right| \leq 2\left\lceil C_{3}\right\rceil
$$

We now turn to the proof of Proposition 4.9.

Proof of Proposition 4.9. The proof is performed in three steps. In the first one, we construct sub- and super-solutions of (4.18) in $\mathbb{R} \times \mathbb{R}$ with good translation invariance properties (see the first two lines of (4.20) ). We next apply Perron's method in order to get a (possibly discontinuous) solution satisfying the same properties. Finally, in Step 3, we prove that if the functions $G_{j}$ do not depend on $\tau$, then we can construct a solution in such a way that it does not depend on $\tau$ either.

Step 1. Global sub- and super-solution.

By Proposition 4.3, we know that the solution $\left(u_{j}, \xi_{j}\right)$ of (2.1), (2.2) with initial data $u_{0}(y)=p y=\xi_{0}(y)$ satisfies on $[0,+\infty) \times \mathbb{R}$,

$$
\left\{\begin{array} { l } 
{ ( u _ { j } ) _ { y } \geq 0 , } \\
{ | u _ { j } ( \tau , y ) - p y - \lambda \tau | \leq C _ { 3 } , } \\
{ | u _ { j } ( \tau , y + y ^ { \prime } ) - u _ { j } ( \tau , y ) - p y ^ { \prime } | \leq 1 , } \\
{ u _ { j + 1 } ( \tau , y ) \geq u _ { j } ( \tau , y ) , }
\end{array} \quad \left\{\begin{array}{l}
\left(\xi_{j}\right)_{y} \geq 0, \\
\left|\xi_{j}(\tau, y)-p y-\lambda \tau\right| \leq C_{3}, \\
\left|\xi_{j}\left(\tau, y+y^{\prime}\right)-\xi_{j}(\tau, y)-p y^{\prime}\right| \leq 1 \\
\xi_{j+1}(\tau, y) \geq \xi_{j}(\tau, y) .
\end{array}\right.\right.
$$

We first construct a sub-solution and a super-solution of (4.18) for $\tau \in \mathbb{R}$ (and not only $\tau \geq 0$ ) that also satisfy the first two lines of (4.20), i.e., satisfy for all $k, l \in \mathbb{Z}$,

$$
U(\tau+k, y)=U\left(\tau, y+\lambda \frac{k}{p}\right) \quad \text { and } \quad U\left(\tau, y+\frac{l}{p}\right)=U(\tau, y)+l .
$$

To do so, we consider for $j \in\{1, \ldots, n\}$ two sequences of functions (indexed by $m \in \mathbb{N}, \quad m \rightarrow \infty)$

$$
u_{j}^{m}(\tau, y)=u_{j}(\tau+m, y)-\lfloor\lambda m\rfloor, \quad \xi_{j}^{m}(\tau, y)=\xi_{j}(\tau+m, y)-\lfloor\lambda m\rfloor
$$

and consider

$$
\begin{aligned}
& \bar{u}_{j}=\limsup _{m \rightarrow+\infty}^{*} u_{j}^{m}, \quad \bar{\xi}_{j}=\limsup _{m \rightarrow+\infty}^{*} \xi_{j}^{m}, \\
& \underline{u}_{j}=\liminf _{m \rightarrow+\infty_{*}} u_{j}^{m}, \quad \underline{\xi}_{j}=\liminf _{m \rightarrow+\infty_{*}} \xi_{j}^{m} .
\end{aligned}
$$

We first remark that thanks to (4.3), all these semi-limits are finite. We also remark that for all $k, l \in \mathbb{Z}$,

$$
\left(\bar{u}_{j}(\tau+k, y-k \lambda / p+l / p)-l, \bar{\xi}_{j}(\tau+k, y-k \lambda / p+l / p)-l\right)
$$

is a sub-solution of (4.18). A similar remark can be made for the super-solutions $\left(\underline{u}_{j}, \underline{\xi}_{j}\right)_{j}$.

Now a way to construct a sub-solution (resp. a super-solution) of (2.1) satisfying (4.24) is to consider

$$
\left\{\begin{array}{l}
\bar{u}_{j}^{\infty}(\tau, y)=\left(\sup _{k, l \in \mathbb{Z}}\left(\bar{u}_{j}(\tau+k, y-k \lambda / p+l / p)-l\right)\right)^{*} \\
\bar{\xi}_{j}^{\infty}(\tau, y)=\left(\sup _{k, l \in \mathbb{Z}}\left(\bar{\xi}_{j}(\tau+k, y-k \lambda / p+l / p)-l\right)\right)^{*}
\end{array}\right.
$$


and

$$
\left\{\begin{array}{l}
\underline{u}_{j}^{\infty}(\tau, y)=\left(\inf _{k, l \in \mathbb{Z}}\left(\underline{u}_{j}(\tau+k, y-k \lambda / p+l / p)-l\right)\right)_{*}, \\
\underline{\xi}_{j}^{\infty}(\tau, y)=\left(\inf _{k, l \in \mathbb{Z}}\left(\underline{\xi}_{j}(\tau+k, y-k \lambda / p+l / p)-l\right)\right)_{*} .
\end{array}\right.
$$

Notice that $\bar{u}_{j}^{\infty}, \underline{u}_{j}^{\infty}, \bar{\xi}_{j}^{\infty}$ and $\underline{\xi}_{j}^{\infty}$ moreover satisfy (4.23) on $\mathbb{R} \times \mathbb{R}$. Therefore we have, in particular,

$$
\bar{u}_{j}^{\infty} \leq \underline{u}_{j}^{\infty}+2\left\lceil C_{3}\right\rceil \quad \text { and } \quad \bar{\xi}_{j}^{\infty} \leq \underline{\xi}_{j}^{\infty}+2\left\lceil C_{3}\right\rceil .
$$

Step 2. Existence by Perron's method.

Applying Perron's method we see that the lowest-* super-solution $\left(\left(u_{j}^{\infty}\right)_{j},\left(\xi_{j}^{\infty}\right)_{j}\right)$ lying above $\left(\left(\bar{u}_{j}^{\infty}\right)_{j},\left(\bar{\xi}_{j}^{\infty}\right)_{j}\right)$ is a (possibly discontinuous) solution of (4.18) on $\mathbb{R} \times \mathbb{R}$ and satisfies

$$
\bar{u}_{j}^{\infty} \leq u_{j}^{\infty} \leq \underline{u}_{j}^{\infty}+2\left\lceil C_{3}\right\rceil \quad \text { and } \quad \bar{\xi}_{j}^{\infty} \leq \xi_{j}^{\infty} \leq \underline{\xi}_{j}^{\infty}+2\left\lceil C_{3}\right\rceil .
$$

We next prove that $u^{\infty}$ satisfies (4.20). For $j \in\{1, \ldots, n\}$, let us consider

$$
\begin{aligned}
& \tilde{u}_{j}^{\infty}(\tau, y)=\left(\inf _{k, l \in \mathbb{Z}}\left(u_{j}^{\infty}(\tau+k, y-k \lambda / p+l / p)-l\right)\right)_{*}, \\
& \tilde{\xi}_{j}^{\infty}(\tau, y)=\left(\inf _{k, l \in \mathbb{Z}}\left(\xi_{j}^{\infty}(\tau+k, y-k \lambda / p+l / p)-l\right)\right)_{*} .
\end{aligned}
$$

By construction the family $\left(\left(\tilde{u}_{j}^{\infty}\right)_{j},\left(\tilde{\xi}_{j}^{\infty}\right)_{j}\right)$ is a super-solution of (4.18) and is again above the sub-solution $\left(\left(\bar{u}_{j}^{\infty}\right)_{j},\left(\bar{\xi}_{j}^{\infty}\right)_{j}\right)$. Therefore from the definition of $\left(\left(u_{j}^{\infty}\right)_{j},\left(\xi_{j}^{\infty}\right)_{j}\right)$, we deduce that

$$
\tilde{u}_{j}^{\infty}=u_{j}^{\infty} \quad \text { and } \quad \tilde{\xi}_{j}^{\infty}=\xi_{j}^{\infty},
$$

which implies that $u_{j}^{\infty}$ and $\xi_{j}^{\infty}$ satisfy (4.24), i.e., the first two equalities of (4.20).

Similarly, we can consider, for $j \in\{1, \ldots, n\}$,

$$
\begin{aligned}
& \hat{u}_{j}^{\infty}(\tau, y)=\left(\inf _{b \in[0,+\infty)} u_{j}^{\infty}(\tau, y+b)\right)_{*}, \\
& \hat{\xi}_{j}^{\infty}(\tau, y)=\left(\inf _{b \in[0,+\infty)} \xi_{j}^{\infty}(\tau, y+b)\right)_{*},
\end{aligned}
$$

which is again a super-solution above the sub-solution $\left(\left(\bar{u}_{j}^{\infty}\right)_{j},\left(\bar{\xi}_{j}^{\infty}\right)_{j}\right)$. Therefore

$$
\hat{u}_{j}^{\infty}=u_{j}^{\infty} \quad \text { and } \quad \hat{\xi}_{j}^{\infty}=\xi_{j}^{\infty},
$$

which implies that $u_{j}^{\infty}$ and $\xi_{j}^{\infty}$ are non-decreasing in $y$, i.e., the third line of (4.20) is satisfied.

Let us now prove that $u_{j}^{\infty}$ and $\xi_{j}^{\infty}$ are non-decreasing in $j$. We consider, for $j \in\{1, \ldots, n\}$,

$$
\begin{aligned}
& \check{u}_{j}^{\infty}(\tau, y)=\left(\inf _{k \geq 0} u_{j+k}^{\infty}(\tau, y)\right)_{*}=\left(\inf _{0 \leq k<n} u_{j+k}^{\infty}(\tau, y)\right)_{*}, \\
& \check{\xi}_{j}^{\infty}(\tau, y)=\left(\inf _{k \geq 0} \xi_{j+k}^{\infty}(\tau, y)\right)_{*}=\left(\inf _{0 \leq k<n} \xi_{j+k}^{\infty}(\tau, y)\right)_{*} .
\end{aligned}
$$


The fact that this is a super-solution uses assumption (A6). Indeed, let us assume that the infimum for $u_{j}$ is reached for the index $k_{u}$ and that the infimum for $\xi_{j}$ is reached for the index $k_{\xi}$. Then, formally, on one hand we have

$$
\begin{aligned}
\left(\check{u}_{j}^{\infty}\right)_{\tau}(\tau, y) & =\alpha_{0}\left(\xi_{j+k_{u}}^{\infty}(\tau, y)-u_{j+k_{u}}^{\infty}(\tau, y)\right) \\
& \geq \alpha_{0}\left(\xi_{j+k_{\xi}}^{\infty}(\tau, y)-u_{j+k_{u}}^{\infty}(\tau, y)\right) \\
& \geq \alpha_{0}\left(\check{\xi}_{j}^{\infty}(\tau, y)-\check{u}_{j}^{\infty}(\tau, y)\right),
\end{aligned}
$$

where we have used the fact that $\xi_{j+k_{u}}^{\infty}(\tau, y) \geq \xi_{j+k_{\xi}}^{\infty}(\tau, y)$. On the other hand, we have

$$
\begin{aligned}
\left(\check{\xi}_{j}^{\infty}\right)_{\tau}(\tau, y)= & G_{j+k_{\xi}}\left(\tau,\left[u^{\infty}(\tau, \cdot)\right]_{j+k_{\xi}}(y), \xi_{j+k_{\xi}}^{\infty}(\tau, y),\right. \\
& \left.\inf _{y^{\prime}}\left(\xi_{j+k_{\xi}}^{\infty}\left(\tau, y^{\prime}\right)-p y^{\prime}\right)+p y-\xi_{j+k_{\xi}}^{\infty}(\tau, y),\left(\xi_{j+k_{\xi}}^{\infty}\right)_{y}\right) \\
\geq & G_{j+k_{\xi}}\left(\tau,\left[\check{u}^{\infty}(\tau, \cdot)\right]_{j+k_{\xi}}(y), \check{\xi}_{j}^{\infty}(\tau, y),\right. \\
& \left.\quad \inf _{y^{\prime}}\left(\check{\xi}_{j}^{\infty}\left(\tau, y^{\prime}\right)-p y^{\prime}\right)+p y-\check{\xi}_{j}^{\infty}(\tau, y),\left(\check{\xi}_{j}^{\infty}\right)_{y}\right) \\
\geq & G_{j+k_{\xi}-1}\left(\tau,\left[\check{u}^{\infty}(\tau, \cdot)\right]_{j+k_{\xi}-1}(y), \check{\xi}_{j}^{\infty}(\tau, y),\right. \\
& \left.\quad \inf _{y^{\prime}}\left(\check{\xi}_{j}^{\infty}\left(\tau, y^{\prime}\right)-p y^{\prime}\right)+p y-\check{\xi}_{j}^{\infty}(\tau, y),\left(\check{\xi}_{j}^{\infty}\right)_{y}\right) \\
\geq & G_{j}\left(\tau,\left[\check{u}^{\infty}(\tau, \cdot)\right]_{j}(y), \check{\xi}_{j}^{\infty}(\tau, y),\right. \\
& \left.\inf _{y^{\prime}}\left(\check{\xi}_{j}^{\infty}\left(\tau, y^{\prime}\right)-p y^{\prime}\right)+p y-\check{\xi}_{j}^{\infty}(\tau, y),\left(\check{\xi}_{j}^{\infty}\right)_{y}\right) .
\end{aligned}
$$

Here we have used the fact that $u_{j+k_{\xi}+k}^{\infty} \geq \check{u}_{j+k_{\xi}+k}^{\infty}$ and $\xi_{j+k_{\xi}}^{\infty}\left(\tau, y^{\prime}\right) \geq \check{\xi}_{j}^{\infty}\left(\tau, y^{\prime}\right)$ joint to the monotonicity assumption of $G$ in the variable $V_{i}$ and $a$ for the first inequality and assumption (A6) joint to the fact that $\check{u}_{j}^{\infty}$ is non-decreasing in $j$ (by construction) for the other inequalities.

We then conclude that $\left(\check{u}_{j}^{\infty}, \check{\xi}_{j}^{\infty}\right)$ is again a super-solution above the sub-solution $\left(\left(\bar{u}_{j}^{\infty}\right)_{j},\left(\bar{\xi}_{j}^{\infty}\right)_{j}\right)$. Therefore

$$
u_{j}^{\infty}=\check{u}_{j}^{\infty} \quad \text { and } \quad \xi_{j}^{\infty}=\check{\xi}_{j}^{\infty},
$$

which implies that $u_{j}^{\infty}$ and $\xi_{j}^{\infty}$ are non-decreasing in $j$, i.e., the fourth line of (4.20) is satisfied.

Finally, the function $\left(\left(u_{j}^{\infty}-\left\lceil C_{3}\right\rceil\right)_{j},\left(\xi_{j}^{\infty}-\left\lceil C_{3}\right\rceil\right)_{j}\right)$ still satisfies (4.20) and also satisfies (4.19).

Step 3. Further properties when the $G_{j}$ are independent on $\tau$.

When the $G_{j}$ do not depend on $\tau$, we can apply Steps 1 and 2 with $k \in \mathbb{Z}$ in (4.25), (4.26) and (4.27) replaced with $k \in \mathbb{R}$. This implies that the hull function $\left(\left(h_{j}\right)_{j},\left(g_{j}\right)_{j}\right)$ does not depend on $\tau$. This ends the proof of the proposition.

Proposition 4.11 (Definition and continuity of the effective Hamiltonian). Consider $p>0$ and assume (A1)-(A6). Then

- there exists a unique real number $\lambda \in \mathbb{R}$ such that there exists a solution $\left(\left(u_{j}^{\infty}\right)_{j},\left(\xi_{j}^{\infty}\right)_{j}\right)$ of (4.18) on $\mathbb{R} \times \mathbb{R}$ such that there exists $C>0$ such that for all $\tau$,

$$
\left|h_{j}(\tau, z)-z\right| \leq C \quad \text { and } \quad\left|g_{j}(\tau, z)-z\right| \leq C,
$$


where the $h_{j}$ and the $g_{j}$ are defined in (4.21) and (4.22); moreover, we can choose $C=2\left\lceil C_{3}\right\rceil$ with $C_{3}$ given in (4.5);

- if $\lambda$ is seen as a function $\bar{G}$ of $p(\lambda=\bar{G}(p))$, then this function $\bar{G}$ : $(0,+\infty) \rightarrow \mathbb{R}$ is continuous.

Before we prove this proposition, let us give the proof of Theorem 1.10 ,

Proof of Theorem [1.10. Just apply Proposition 4.11 with $G=F$.

Proof of Proposition 4.11. The proof follows classical arguments. However, we present it for the reader's convenience. The proof is divided in two steps.

Step 1. Uniqueness of $\lambda$.

Given some $p \in(0,+\infty)$, assume that there exist $\lambda_{1}, \lambda_{2} \in \mathbb{R}$ with their corresponding hull functions $\left(\left(h_{j}^{1}\right)_{j},\left(g_{j}^{1}\right)_{j}\right),\left(\left(h_{j}^{2}\right)_{j},\left(g_{j}^{2}\right)_{j}\right)$. Then define for $i=1,2$, $j \in\{1, \ldots, n\}$,

$$
u_{j}^{i}(\tau, y)=h_{j}^{i}\left(\tau, \lambda_{i} \tau+p y\right) \quad \text { and } \quad \xi_{j}^{i}(\tau, y)=g_{j}^{i}\left(\tau, \lambda_{i} \tau+p y\right)
$$

which are both solutions of equation (2.1) on $[0,+\infty) \times \mathbb{R}$. By Corollary 4.10, we know that $h_{j}$ and $g_{j}$ satisfy (4.28). Then we have with $C=2\left\lceil C_{3}\right\rceil$

$$
u_{j}^{1}(0, y) \leq u_{j}^{2}(0, y)+2 C \quad \text { and } \quad \xi_{j}^{1}(0, y) \leq \xi_{j}^{2}(0, y)+2 C,
$$

which implies (from the comparison principle) for all $(\tau, y) \times[0,+\infty) \times \mathbb{R}$,

$$
u_{j}^{1}(\tau, y) \leq u_{j}^{2}(\tau, y)+2 C \quad \text { and } \quad \xi_{j}^{1}(\tau, y) \leq \xi_{j}^{2}(\tau, y)+2 C .
$$

Using the fact that $h_{j}^{i}(\tau+1, z)=h_{j}^{i}(\tau, z)$ and $g_{j}^{i}(\tau+1, z)=g_{j}^{i}(\tau, z)$, we deduce that for $\tau=k \in \mathbb{N}$ and $y=0$ we have

$$
h_{j}^{1}\left(0, \lambda_{1} k\right) \leq h_{j}^{2}\left(0, \lambda_{2} k\right)+2 C \quad \text { and } \quad g_{j}^{1}\left(0, \lambda_{1} k\right) \leq g_{j}^{2}\left(0, \lambda_{2} k\right)+2 C,
$$

which implies by (4.28),

$$
\lambda_{1} k \leq \lambda_{2} k+4 C .
$$

Because this is true for any $k \in \mathbb{N}$, we deduce that

$$
\lambda_{1} \leq \lambda_{2} .
$$

The reverse inequality is obtained by exchanging $\left(\left(h_{j}^{1}\right)_{j},\left(g_{j}^{1}\right)_{j}\right)$ and $\left(\left(h_{j}^{2}\right)_{j},\left(g_{j}^{2}\right)_{j}\right)$. We finally deduce that $\lambda_{1}=\lambda_{2}$, which proves the uniqueness of the real $\lambda$, that we call $\bar{G}(p)$.

Step 2. Continuity of the map $p \mapsto \bar{G}(p)$.

Let us consider a sequence $\left(p_{m}\right)_{m}$ such that $p_{m} \rightarrow p>0$. Let $\lambda_{m}=\bar{G}\left(p_{m}\right)$ and $\left(\left(h_{j}^{m}\right)_{j},\left(g_{j}^{m}\right)_{j}\right)$ be the corresponding hull functions. From Corollary 4.10, we can choose these hull functions such that for $j \in\{1, \ldots, n\}$,

$$
\left|h_{j}^{m}(\tau, z)-z\right| \leq 2\left\lceil C_{3}\right\rceil \text { and }\left|g_{j}^{m}(\tau, z)-z\right| \leq 2\left\lceil C_{3}\right\rceil,
$$

and we have

$$
\left|\lambda_{m}\right| \leq C_{4}
$$

where we recall that $C_{4}$ is defined in (4.5). Recall that both $C_{3}$ and $C_{4}$ depend on $p_{m}$ but can be bounded for $p_{m}$ in a neighbourhood of $p$. We deduce in particular that there exists a constant $C_{5}>0$ such that

$$
\left|h_{j}^{m}(\tau, z)-z\right| \leq C_{5}, \quad\left|g_{j}^{m}(\tau, z)-z\right| \leq C_{5} \quad \text { and } \quad\left|\lambda_{m}\right| \leq C_{5} .
$$


Let us consider a limit $\lambda_{\infty}$ of $\left(\lambda_{m}\right)_{m}$, and let us define

$$
\bar{h}_{j}=\limsup _{m \rightarrow+\infty} h_{j}^{m} \text { and } \quad \bar{g}_{j}=\limsup _{m \rightarrow+\infty}^{*} g_{j}^{m} .
$$

This family of functions $\left(\left(\bar{h}_{j}\right)_{j},\left(\bar{g}_{j}\right)_{j}\right)$ is such that the family

$$
\left(\left(\bar{u}_{j}(\tau, y)\right)_{j},\left(\bar{\xi}_{j}(\tau, y)\right)_{j}\right)=\left(\left(\bar{h}_{j}\left(\tau, \lambda_{\infty} \tau+p y\right)\right)_{j},\left(\bar{g}_{j}\left(\tau, \lambda_{\infty} \tau+p y\right)\right)_{j}\right)
$$

is a sub-solution of (4.18) on $\mathbb{R} \times \mathbb{R}$. On the other hand, if $\left(\left(h_{j}\right)_{j},\left(g_{j}\right)_{j}\right)$ denotes the hull function associated with $p$ and $\lambda=\bar{G}(p)$, then

$$
\left(\left(u_{j}(\tau, y)\right)_{j},\left(\xi_{j}(\tau, y)\right)_{j}\right)=\left(\left(h_{j}(\tau, \lambda \tau+p y)\right)_{j},\left(g_{j}(\tau, \lambda \tau+p y)\right)_{j}\right)
$$

is a solution of (4.18) on $\mathbb{R} \times \mathbb{R}$. Finally, as in Step 1, we conclude that

$$
\lambda_{\infty} \leq \lambda .
$$

Similarly, considering

$$
\underline{h}_{j}=\liminf _{m \rightarrow+\infty} h_{j}^{m} \quad \text { and } \quad \underline{g}_{j}=\liminf _{m \rightarrow+\infty}{ }_{*} g_{j}^{m},
$$

we can show that

$$
\lambda_{\infty} \geq \lambda .
$$

Therefore $\lambda_{\infty}=\lambda$, and this proves that $\bar{G}\left(p_{m}\right) \rightarrow \bar{G}(p)$. The continuity of the map $p \mapsto \bar{G}(p)$ follows, and this ends the proof of the proposition.

\section{Construction of Lipschitz Continuous approximate hull FunCtions}

When proving the convergence theorem, Theorem 1.5, we explained that, on the one hand, it is necessary to deal with hull functions $(h, g)=\left(\left(h_{j}(\tau, z)\right)_{j},\left(g_{j}(\tau, z)\right)_{j}\right)$ that are uniformly continuous in $z$ (uniformly in $\tau$ and $j$ ) in order to apply Evans' perturbed test function method. On the other hand, given some $p>0$, we also know some Hamiltonians $F_{j}$, with corresponding effective Hamiltonian $\bar{F}(p)$, such that every corresponding hull function $h_{j}$ is necessarily discontinuous in $z$ for $\alpha_{0}=+\infty$ (see [1, 10]). Recall that a hull function $(h, g)$ solves in particular

$$
\left\{\begin{array}{l}
\left(h_{j}\right)_{\tau}+\lambda\left(h_{j}\right)_{z}=\alpha_{0}\left(g_{j}-h_{j}\right) \\
\left(g_{j}\right)_{\tau}+\lambda\left(g_{j}\right)_{z}=2 F_{j}\left(\tau,[h(\tau, \cdot)]_{j, m}\right)+\alpha_{0}\left(h_{j}-g_{j}\right)
\end{array}\right.
$$

with $\lambda=\bar{F}(p)$ and

$$
h_{j+n}(\tau, z)=h_{j}(\tau, z+p), g_{j+n}(\tau, z)=g_{j}(\tau, z+p) .
$$

We overcome this difficulty as in [10] (see also [11, 14, 15]).

We build approximate Hamiltonians $G^{\delta}$ with corresponding effective Hamiltonians $\lambda^{\delta}=\bar{G}^{\delta}(p)$ and corresponding hull functions $\left(h^{\delta}, g^{\delta}\right)$ such that

$$
\left\{\begin{array}{l}
\left(h_{j}^{\delta}, g_{j}^{\delta}\right) \quad \text { is Lipschitz continuous with respect to } z \text { uniformly in } \tau \text { and } j, \\
\bar{G}(p) \rightarrow \bar{F}(p) \text { as } \delta \rightarrow 0, \\
\left(h^{\delta}, g^{\delta}\right) \text { is a sub-/super-solution of (5.1). }
\end{array}\right.
$$

We will show that it is enough to choose for $\delta \geq 0$ that

$$
G_{j}^{\delta}(\tau, V, r, a, q)=2 F_{j}(\tau, V)+\alpha_{0}\left(V_{0}-r\right)+\delta\left(a_{0}+a\right) q^{+},
$$

with $a_{0} \in \mathbb{R}$ (in fact, we will consider $a_{0}= \pm 1$ ).

We have the following variant of Corollary 4.10 . 
Proposition 5.1 (Existence of Lipschitz continuous approximate hull functions). Assume (A1)-(A3). Given $p>0,0<\delta \leq 1$ and $a_{0} \in \mathbb{R}$, there exists a family of Lipschitz continuous functions $\left(\left(h_{j}\right)_{j},\left(g_{j}\right)_{j}\right)$ satisfying, for $j \in\{1, \ldots, n\}$,

$$
\left\{\begin{array} { l } 
{ h _ { j } ( \tau , z + 1 ) = h _ { j } ( \tau , z ) + 1 , } \\
{ h _ { j } ( \tau + 1 , z ) = h _ { j } ( \tau , z ) , } \\
{ 0 \leq ( h _ { j } ) _ { z } \leq 1 + \frac { 2 L _ { F } } { p \delta } , }
\end{array} \quad \left\{\begin{array}{l}
g_{j}(\tau, z+1)=g_{j}(\tau, z)+1, \\
g_{j}(\tau+1, z)=g_{j}(\tau, z), \\
0 \leq\left(g_{j}\right)_{z} \leq 1+\frac{2 L_{F}}{p \delta},
\end{array}\right.\right.
$$

and there exists $\lambda \in \mathbb{R}$ such that

(5.4)

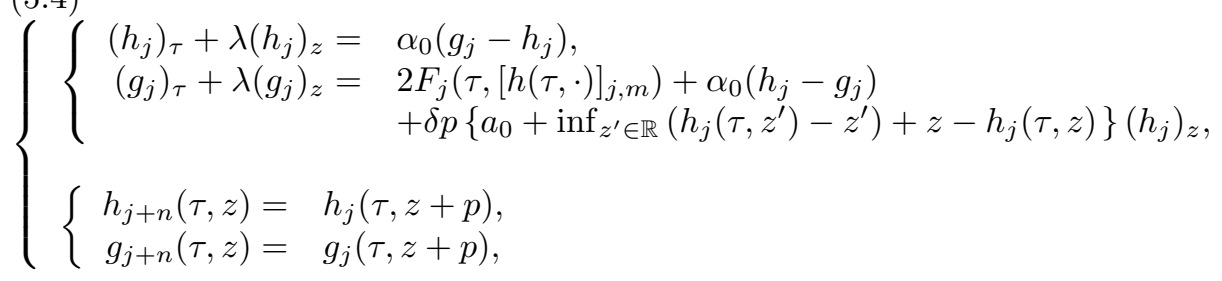

and for all $\tau, z, z^{\prime} \in \mathbb{R}$,

$$
\left|h_{j}\left(\tau, z^{\prime}\right)-z^{\prime}+z-h_{j}(\tau, z)\right| \leq 1 \quad \text { and } \quad\left|g_{j}\left(\tau, z^{\prime}\right)-z^{\prime}+z-g_{j}(\tau, z)\right| \leq 1 .
$$

Moreover, there exists a constant $C_{4}>0$ defined in (4.5) such that

$$
|\lambda| \leq C_{4}
$$

and for all $(\tau, z) \in \mathbb{R} \times \mathbb{R}$,

$$
\begin{aligned}
& |h(\tau, z)-z| \leq C\left(C_{4}, p, \alpha_{0}, \delta\left|a_{0}\right| p\right), \\
& |g(\tau, z)-z| \leq C\left(C_{4}, p, \alpha_{0}, \delta\left|a_{0}\right| p\right) .
\end{aligned}
$$

Moreover, when the $F_{j}$ do not depend on $\tau$, we can choose the hull function $\left(\left(h_{j}\right)_{j},\left(g_{j}\right)_{j}\right)$ such that it does not depend on $\tau$ either.

Proof of Proposition 5.1. The construction follows the one made in Proposition 4.3 and Proposition 4.9. However, Proposition 4.9 has to be adapted. Indeed, since we want to construct a Lipschitz continuous function with a precise Lipschitz estimate, we do not want to use Perron's method. This is the reason why here we can use a space-time Lipschitz estimate of $\left(\left(u_{j}\right),\left(\xi_{j}\right)\right)$ to get enough compacity to pass to the limit.

The space Lipschitz estimate comes from Proposition 4.1. The time Lipschitz estimate of the $u_{j}$ 's follows from Lemma 4.6 and the equation satisfied by $u_{j}$. The time Lipschitz estimate of the $\xi_{j}$ 's is obtained in the same way, using the fact that we can bound the right hand side of the equation satisfied by $\xi_{j}$. Indeed, one can use the space oscillation estimate of $u$ to bound $F\left(t,[u(t, \cdot)]_{j, m}(x)\right.$ ) (as we did in (4.11)-(4.12) ) and use Lemma 4.6 and Proposition 4.1 to bound remaining terms.

We finally have

Proposition 5.2 (Sub- and super- Lipschitz continuous hull functions). We consider $0<\delta \leq 1$ and the Lipschitz continuous hull function obtained in Proposition 
5.1 for $a_{0}= \pm 1$, that we call $\left(\left(h_{j}^{\delta, \pm}\right)_{j},\left(g_{j}^{\delta, \pm}\right)_{j}\right)$, and the corresponding value $\lambda^{\delta, \pm}$ of the effective Hamiltonian. Then we have

$$
\begin{aligned}
\left(h_{j}^{\delta,+}\right)_{\tau}+\lambda^{\delta,+}\left(h_{j}^{\delta,+}\right)_{z} & =\alpha_{0}\left(g_{j}^{\delta,+}-h_{j}^{\delta,+}\right) \\
\left(g_{j}^{\delta,+}\right)_{\tau}+\lambda^{\delta,+}\left(g_{j}^{\delta,+}\right)_{z} & \geq 2 F_{j}\left(\tau,\left[h^{\delta,+}(\tau, \cdot)\right]_{j, m}\right)+\alpha_{0}\left(h_{j}^{\delta,+}-g_{j}^{\delta,+}\right)
\end{aligned}
$$

and

$$
\lambda \leq \lambda^{\delta,+} \rightarrow \lambda \quad \text { as } \quad \delta \rightarrow 0
$$

and

$$
\begin{aligned}
\left(h_{j}^{\delta,-}\right)_{\tau}+\lambda^{\delta,-}\left(h_{j}^{\delta,-}\right)_{z} & =\alpha_{0}\left(g_{j}^{\delta,-}-h_{j}^{\delta,-}\right) \\
\left(g_{j}^{\delta,-}\right)_{\tau}+\lambda^{\delta,-}\left(g_{j}^{\delta,-}\right)_{z} & \geq 2 F_{j}\left(\tau,\left[h^{\delta,-}(\tau, \cdot)\right]_{j, m}\right)+\alpha_{0}\left(h_{j}^{\delta,-}-g_{j}^{\delta,-}\right)
\end{aligned}
$$

and

$$
\lambda \geq \lambda^{\delta,-} \rightarrow \lambda \quad \text { as } \quad \delta \rightarrow 0
$$

where $\lambda=\bar{F}(p)$.

Proof of Proposition 5.2. Inequalities $\pm \lambda^{\delta, \pm} \geq \pm \lambda$ follow from the comparison principle. Recall that bounds (5.6) and (5.7) on $\lambda^{\delta, \pm}$ and $h_{j}^{\delta, \pm}$ are uniform as $\delta$ goes to zero. Hence the convergence $\lambda^{\delta, \pm} \rightarrow \lambda$ holds true as $\delta \rightarrow 0$. Indeed, it suffices to adapt Step 2 of the proof of Proposition 4.11 .

\section{Qualitative Properties of the effective Hamiltonian}

Proof of Theorem 1.11. We recall that we have hull function solutions $\left(\left(h_{j}\right)_{j},\left(g_{j}\right)_{j}\right)$ of

$$
\left\{\begin{array}{l}
\left(h_{j}\right)_{\tau}+\lambda\left(h_{j}\right)_{z}=\alpha_{0}\left(g_{j}-h_{j}\right) \\
\left(g_{j}\right)_{\tau}+\lambda\left(g_{j}\right)_{z}=2 L+2 F\left(\tau,[h(\tau, \cdot)]_{j, m}(z)\right)+\alpha_{0}\left(h_{j}-g_{j}\right)
\end{array}\right.
$$

with $\lambda=\bar{F}(L, p)$.

The continuity of the map $(L, p) \mapsto \bar{F}(L, p)$ is easily proved as in Step 2 of the proof of Proposition 4.11.

(i) Bound

This is a straightforward adaptation of the proof of (4.13).

(ii) Monotonicity in $L$

The monotonicity of the map $L \mapsto \bar{F}(L, p)$ follows from the comparison principle on

$$
\left(\left(u_{j}(\tau, y)=h_{j}(\tau, \lambda \tau+p y)\right)_{j},\left(\xi_{j}(\tau, y)=g_{j}(\tau, \lambda \tau+p y)\right)_{j}\right)
$$

where $\left(\left(h_{j}\right)_{j},\left(g_{j}\right)_{j}\right)$ is the hull function and $\lambda=\bar{F}(L, p)$.

\section{Appendix A. An alternative proof of Proposition 4.1}

In this section, we give an alternative proof of Proposition 4.1 Here we adapt the method we used in $[10$ and provide complementary details. 
A.1. Explanation of the estimate of Proposition 4.1. In this section, we formally explain how we derive the estimate obtained in Proposition 4.1

We can adapt the corresponding proof from [10]. For all $\eta \geq 0$, we consider the following Cauchy problem:

$$
\left\{\begin{array}{c}
\left\{\begin{array}{r}
\left(u_{j}\right)_{\tau}=\alpha_{0}\left(\xi_{j}-u_{j}\right) \\
\left(\xi_{j}\right)_{\tau}=G_{j}^{\delta}\left(\tau,[u(\tau, \cdot)]_{j, m}, \xi_{j}(\tau, y), \inf _{y^{\prime} \in \mathbb{R}}\left(\xi_{j}\left(\tau, y^{\prime}\right)-p y^{\prime}\right)\right. \\
\left.\quad+p y-\xi_{j}(\tau, y),\left(\xi_{j}\right)_{y}\right)+\eta\left(\xi_{j}\right)_{y y},
\end{array}\right. \\
\left\{\begin{array}{r}
u_{j+n}(\tau, y)=u_{j}(\tau, y+1) \\
\xi_{j+n}(\tau, y)=\xi_{j}(\tau, y+1)
\end{array}\right. \\
\left\{\begin{aligned}
, u_{j}(0, y)=p\left(y+\frac{j}{n}\right) \\
\xi_{j}(0, y)=p\left(y+\frac{j}{n}\right)
\end{aligned}\right.
\end{array}\right.
$$

where $G_{j}^{\delta}$ is given by

$$
G_{j}^{\delta}(\tau, V, r, a, q)=2 F_{j}(\tau, V)+\alpha_{0}\left(V_{0}-r\right)+\delta\left(a_{0}+a\right) q
$$

(we remark that this is not exactly the function given by (5.2) ). It is convenient to introduce the modified Hamiltonian

$$
\tilde{F}_{i}\left(\tau, V_{-m}, \ldots, V_{m}\right)=2 F_{i}\left(\tau, V_{-m}, \ldots, V_{m}\right)+\alpha_{0} V_{0}
$$

so that

$$
G_{j}^{\delta}\left(\tau, V_{-m}, \ldots, V_{m}, r, a, q\right)=\tilde{F}_{j}\left(\tau, V_{-m}, \ldots, V_{m}\right)-\alpha_{0} r+\delta\left(a_{0}+a\right) q .
$$

Hence, the Lipschitz constant of $\tilde{F}_{j}(\tau, V)$ with respect to $V$ is $\tilde{K}_{1}=2 L_{F}+\alpha_{0}$.

Case A. $\eta>0$ and $F_{j} \in C^{1}$.

For $\eta>0$, it is possible to show that there exists a unique solution $\left(\left(u_{j}\right)_{j},\left(\xi_{j}\right)_{j}\right)$ of A.1) in $\left(C^{2+\alpha, 1+\alpha}\right)^{2 n}$ for any $\alpha \in(0,1)$. We will give the main idea of this existence result in the next subsection.

Step 1. Bound from below on the gradient.

Then, if we define $\zeta_{j}=\left(\xi_{j}\right)_{y}$ and $v_{j}=\left(u_{j}\right)_{y}$, we can derive the previous equation in order to get the following one:

$$
\left\{\begin{aligned}
\left(v_{j}\right)_{\tau}= & \alpha_{0}\left(\zeta_{j}-v_{j}\right) \\
\left(\zeta_{j}\right)_{\tau}-\eta\left(\zeta_{j}\right)_{y y}= & \left(\tilde{F}_{j}\right)_{V}^{\prime}\left(\tau,[u(\tau, \cdot)]_{j, m}(y)\right) \cdot[v(\tau, \cdot)]_{j, m}(y)-\alpha_{0} \zeta_{j}-\delta\left(\zeta_{j}-p\right) \zeta_{j} \\
& +\delta\left(a_{0}+\inf _{y^{\prime} \in \mathbb{R}}\left(\xi_{j}\left(\tau, y^{\prime}\right)-p y^{\prime}\right)+p y-\xi_{j}(\tau, y)\right)\left(\zeta_{j}\right)_{y} \\
v_{j+n}(\tau, y)= & v_{j}(\tau, y+1) \\
\zeta_{j+n}(\tau, y)= & \zeta_{j}(\tau, y+1) \\
v_{j}(0, y)= & \zeta_{j}(0, y)=p
\end{aligned}\right.
$$

Let us now define

$$
\underline{m}_{v}(\tau)=\inf _{j \in\{1, \ldots, n\}} \inf _{y \in \mathbb{R}} v_{j}(\tau, y) \quad \text { and } \quad \underline{m}_{\zeta}(\tau)=\inf _{j \in\{1, \ldots, n\}} \inf _{y \in \mathbb{R}} \zeta_{j}(\tau, y) .
$$

Then we have in the viscosity sense

$$
\left\{\begin{array}{l}
\left(\underline{m}_{v}\right)_{\tau} \geq \alpha_{0}\left(\underline{m}_{\zeta}-\underline{m}_{v}\right) \\
\left(\underline{m}_{\zeta}\right)_{\tau} \geq \tilde{L}_{F} \min \left(0, \underline{m}_{v}\right)-\alpha_{0} \underline{m}_{\zeta}-\delta\left(\underline{m}_{\zeta}-p\right) \underline{m}_{\zeta} \\
\underline{\underline{m}}_{v}(0)=\underline{m}_{\zeta}(0)=p>0
\end{array}\right.
$$


where we have used the monotonicity assumptions (A2) and (A3) to get the term $\tilde{L}_{F} \min \left(0, \underline{m}_{v}\right)$ with $\tilde{L}_{F}=2 L_{F}+\alpha_{0}$. The fact that $(0,0)$ is a sub-solution of this monotone system of ODEs implies that, for $j \in\{1, \ldots, n\}$,

$$
v_{j} \geq \underline{m}_{v} \geq 0 \quad \text { and } \quad \zeta_{j} \geq \underline{m}_{\zeta} \geq 0 .
$$

In particular, we see that $(u, \xi)$ is a solution of (A.1) with $G_{j}^{\delta}$ given by (5.2).

Step 2. Bound from above on the gradient.

Similarly, we define

$$
\bar{m}_{\zeta}(\tau)=\sup _{j \in\{1, \ldots, n\}} \sup _{y \in \mathbb{R}} \zeta_{j}(\tau, y) \quad \text { and } \quad \bar{m}_{v}(\tau)=\sup _{j \in\{1, \ldots, n\}} \sup _{y \in \mathbb{R}} v_{j}(\tau, y) .
$$

Then we have in the viscosity sense

$$
\left\{\begin{aligned}
\left(\bar{m}_{v}\right)_{\tau} & \leq \alpha_{0}\left(\bar{m}_{\zeta}-\bar{m}_{v}\right) \\
\left(\bar{m}_{\zeta}\right)_{\tau} & \leq\left(2 L_{F}\right) \bar{m}_{v}+\alpha_{0}\left(\bar{m}_{v}-\bar{m}_{\zeta}\right)-\delta\left(\bar{m}_{\zeta}-p\right) \bar{m}_{\zeta}, \\
\bar{m}_{v}(0) & =\bar{m}_{\zeta}(0)=p>0
\end{aligned}\right.
$$

where we have used Step 1 to ensure that $v_{j} \geq \underline{m}_{v} \geq 0$ for $j \in\{1, \ldots, n\}$. The constant function $\left(p+\left(2 L_{F}\right) \delta^{-1}\right)$ (for both components) is a super-solution of the previous monotone system of ODEs. Hence, the proof is complete in Case A.

Case B. $\eta=0$ and $F$ general.

We can use an approximation argument as in [10.

This ends the proof of the proposition.

A.2. Proof of the existence of a regular solution of (A.1). We just give the main idea.

It can be useful to remark that $u_{j+l}$ can be rewritten as follows: for all $l \in$ $\{-m, \ldots, m\}$,

$$
u_{j+l}(\tau, y)=p \cdot(y+(j+l) / n) e^{-\alpha_{0} \tau}+\alpha_{0} \int_{0}^{\tau} e^{\alpha_{0}(s-\tau)} \xi_{j+l}(s, y) d s .
$$

We set $v_{j}(\tau, y)=\xi_{j}(\tau, y)-p y$. Then $\left(v_{j}\right)_{j}$ is a solution of

$$
\left\{\begin{array}{c}
\left(v_{j}\right)_{t}-\eta\left(v_{j}\right)_{y y}=\bar{F}_{j}\left(t,[v(\tau, \cdot)+p \cdot]_{j, m}(y)\right) \\
\quad+\delta\left(1+\inf _{y^{\prime}}\left(v\left(\tau, y^{\prime}\right)\right)-v(\tau, y)\right)\left(v_{y}+p\right), \\
v_{j+n}(\tau, y)=v_{j}(\tau, y+1)+p \\
v_{j}(0, y)=p\left(\frac{j}{n}\right),
\end{array}\right.
$$

where $\bar{F}_{j}\left[\tau,[\xi(\tau, \cdot)]_{j, m}(y)\right]=2 F_{j}\left(\tau,[u(\tau, \cdot)]_{j, m}(y)\right)+\alpha_{0} u_{j}(\tau, y)-\xi_{j}(\tau, y)$ with $u$ given by A.3 as a function of the time integral of $\xi$. Since we attempt to get $\xi_{j}\left(\tau, y+\frac{1}{p}\right)=\xi_{j}(\tau, y)+1$, we will look for functions $v_{j}$ which are periodic of period $\frac{1}{p}$. The basic idea is to use a fixed point argument. First, we "regularize" the right hand side of (A.4) by considering for some given $K>0$,

$$
\begin{aligned}
\mathcal{F}_{K, j}(\tau, v)= & T_{K}^{0}\left(\bar{F}_{j}\left(\tau,[v(\tau, \cdot)+p \cdot]_{j, m}(y)\right)\right) \\
& +\delta\left(1+T_{K}^{1}\left(\inf _{y^{\prime}}\left(v\left(\tau, y^{\prime}\right)\right)-v(\tau, y)\right)\right)\left(T_{K}^{3}\left(v_{y}+p\right)\right),
\end{aligned}
$$


where $T_{K}^{i} \in C_{b}^{\infty}$ are truncature functions. In particular, $\mathcal{F}_{K, j}(\tau, \cdot) \in W^{1, \infty}$ uniformly in $\tau \in[0,+\infty)$, and so for all $q>1$, there exists a solution $w=\left(w_{j}\right)_{j}=$ $A(v) \in W^{2,1 ; q}\left([0, T] \times\left[0, \frac{1}{p}\right)\right)$ of

$$
\left(w_{j}\right)_{t}-\eta\left(w_{j}\right)_{y y}=\mathcal{F}_{K, j}(v) .
$$

Now, we want to show that the operator $A$ is a contraction. Let

$$
v_{1}, v_{2} \in W^{2,1 ; q}\left([0, T] \times\left[0, \frac{1}{p}\right)\right) .
$$

Standard parabolic estimates show that

$$
\begin{aligned}
& \left|A_{j}\left(v_{1}\right)-A_{j}\left(v_{2}\right)\right|_{W^{2,1 ; q}\left([0, T] \times\left[0, \frac{1}{p}\right)\right)} \\
& \leq C\left|\mathcal{F}_{K, j}\left(\tau, v_{1}\right)-\mathcal{F}_{K, j}\left(\tau, v_{2}\right)\right|_{L^{q}\left([0, T] \times\left[0, \frac{1}{p}\right)\right)} \\
& \leq C\left(\left|v_{2}-v_{1}\right|_{L^{q}\left([0, T] \times\left[0, \frac{1}{p}\right)\right)}+\left|\inf \left(v_{2}\right)-v_{2}-\left(\inf \left(v_{1}\right)-v_{1}\right)\right|_{L^{q}\left([0, T] \times\left[0, \frac{1}{p}\right)\right)}\right. \\
& \left.\quad \quad \quad \quad\left|\left(v_{2}-v_{1}\right)_{y}\right|_{L^{q}\left([0, T] \times\left[0, \frac{1}{p}\right)\right)}\right) \\
& \quad \leq C T^{\beta}\left|v_{2}-v_{1}\right|_{W^{2,1 ; q}\left([0, T] \times\left[0, \frac{1}{p}\right)\right)}
\end{aligned}
$$

for some $\beta>0$ (see [20, 13]).

Sobolev embedding and parabolic regularity theory in Holder's spaces implies the existence for $T$ small enough of a solution $w_{j} \in C^{2+\alpha, \frac{2+\alpha}{2}}$.

While we have smooth solutions below the truncature, we can apply the arguments of Subsection A.1 and get estimates on the gradient of the solution which ensures that the solution is indeed below the truncature. Finally, a posteriori, the truncature can be completely removed because of our estimate on the gradient of the solution.

\section{ACKNOWLEDGEMENTS}

This work was partially supported by the ANR-funded project "MICA" (20062010). The second author was also partially supported by the ANR-funded project "Kam Faible" (2008-2012).

\section{REFERENCES}

[1] S. Aubry, The twist map, the extended Frenkel-Kontorova model and the devil's staircase, Phys. D, 7 (1983), pp. 240-258. Order in chaos (Los Alamos, N.M., 1982). MR719055 (85d:58046)

[2] C. Baesens and R. S. MacKay, A novel preserved partial order for cooperative networks of units with overdamped second order dynamics, and application to tilted Frenkel-Kontorova chains, Nonlinearity, 17 (2004), pp. 567-580. MR2039059 (2004k:34073)

[3] G. Barles, Solutions de viscosité des équations de Hamilton-Jacobi, vol. 17 of Mathématiques \& Applications (Berlin) [Mathematics \& Applications], Springer-Verlag, Paris, 1994. MR.1613876 (2000b:49054)

[4] O. M. Braun and Y. S. Kivshar, The Frenkel-Kontorova model, Texts and Monographs in Physics, Springer-Verlag, Berlin, 2004. Concepts, methods, and applications. MR2035039 (2004k:82001)

[5] F. Camilli, O. Ley, and P. Loreti, Homogenization of monotone systems of HamiltonJacobi equations, ESAIM Control Optim. Calc. Var., 16 (2010), pp. 58-76. MR2598088 (2011d:35035)

[6] I. Capuzzo-Dolcetta and L. C. Evans, Optimal switching for ordinary differential equations, SIAM J. Control Optim., 22 (1984), pp. 143-161. MR728678 (85b:49043) 
[7] M. G. Crandall, H. Ishii, and P.-L. Lions, User's guide to viscosity solutions of second order partial differential equations, Bull. Amer. Math. Soc. (N.S.), 27 (1992), pp. 1-67. MR.1118699 (92j:35050)

[8] R. de la Llave, KAM theory for equilibrium states in 1-D statistical mechanics models, Ann. Henri Poincaré, 9 (2008), pp. 835-880. MR2438500 (2009f:82007)

[9] L. C. Evans, The perturbed test function method for viscosity solutions of nonlinear PDE, Proc. Roy. Soc. Edinburgh Sect. A, 111 (1989), pp. 359-375. MR.1007533 (91c:35017)

[10] N. Forcadel, C. Imbert, and R. Monneau, Homogenization of fully overdamped FrenkelKontorova models, J. Differential Equations, 246 (2009), pp. 1057-1097. MR2474586 (2009j:35022)

[11] N. Forcadel, C. Imbert, and R. Monneau, Homogenization of some particle systems with twobody interactions and of the dislocation dynamics, Discrete Contin. Dyn. Syst., 23 (2009), pp. 785-826. MR:2461827 (2010h:35021)

[12] B. Hu, W.-X. Qin, and Z. Zheng, Rotation number of the overdamped Frenkel-Kontorova model with ac-driving, Phys. D, 208 (2005), pp. 172-190. MR2167606 (2006e:37146)

[13] H. Ibrahim, M. Jazar, and R. Monneau, Dynamics of dislocation densities in a bounded channel. II. Existence of weak solutions to a singular Hamilton-Jacobi/parabolic strongly coupled system, Comm. Partial Differential Equations, 34 (2009), pp. 889-917. MR2560304 (2010k:35062)

[14] C. Imbert and R. Monneau, Homogenization of first-order equations with $(u / \epsilon)$-periodic Hamiltonians. I. Local equations, Arch. Ration. Mech. Anal., 187 (2008), pp. 49-89. MR2358335 (2010e:35029)

[15] C. Imbert, R. Monneau, and E. Rouy, Homogenization of first order equations with $(u / \epsilon)$ periodic Hamiltonians. II. Application to dislocations dynamics, Comm. Partial Differential Equations, 33 (2008), pp. 479-516. MR2398239(2010e:35030)

[16] H. Ishii, Perron's method for monotone systems of second-order elliptic partial differential equations, Differential Integral Equations, 5 (1992), pp. 1-24. MR1141724 (92h:35071)

[17] H. Ishii and S. Koike, Viscosity solutions for monotone systems of second-order elliptic PDEs, Comm. Partial Differential Equations, 16 (1991), pp. 1095-1128. MR.1116855 (92h:35066)

[18] G. James and Y. Sire, Travelling breathers with exponentially small tails in a chain of nonlinear oscillators, Comm. Math. Phys., 257 (2005), pp. 51-85. MR2163569 (2006k:37204)

[19] T. Kato, Perturbation theory for linear operators, Classics in Mathematics, Springer-Verlag, Berlin, 1995. Reprint of the 1980 edition. MR.1335452 (96a:47025)

[20] O. A. Ladyženskaja, V. A. Solonnikov, and N. N. Ural'ceva, Linear and quasilinear equations of parabolic type, Translated from the Russian by S. Smith. Translations of Mathematical Monographs, Vol. 23, American Mathematical Society, Providence, R.I., 1967. MR 0241822 $(39: 3159 \mathrm{~b})$

[21] S. M. Lenhart, Viscosity solutions for weakly coupled systems of first-order partial differential equations, J. Math. Anal. Appl., 131 (1988), pp. 180-193. MR934440 (89m:35025)

CEREmade, UMr CNRS 7534, Université Paris-Dauphine, Place de Lattre de TasSigny, 75775 Paris Cedex 16, France

CNRS, UmR 8050, Centre de Mathématiques, Université Paris-Est Créteil, Val de Marne P3, 61 Avenue du Général de Gaulle, 94010 Créteil Cedex France

Cermics, Universite Paris-Est, Ecole des ponts, 6-8 avenue Blaise Pascal, 77455 Marne la Vallee Cedex 2, France 